\title{
Periostin advances atherosclerotic and rheumatic cardiac valve degeneration by inducing angiogenesis and MMP production in humans and rodents
}

\author{
Daihiko Hakuno,1,2 Naritaka Kimura,1,3 Masatoyo Yoshioka, ${ }^{1}$ \\ Makio Mukai, ${ }^{4}$ Tokuhiro Kimura, ${ }^{5}$ Yasunori Okada, ${ }^{5}$ Ryohei Yozu, ${ }^{3}$ \\ Chisa Shukunami, ${ }^{6}$ Yuji Hiraki, ${ }^{6}$ Akira Kudo, ${ }^{7}$ Satoshi Ogawa, ${ }^{2}$ and Keiichi Fukuda ${ }^{1}$ \\ 1Department of Regenerative Medicine and Advanced Cardiac Therapeutics, ${ }^{2}$ Cardiology Division, Department of Internal Medicine, \\ ${ }^{3}$ Department of Cardiovascular Surgery, ${ }^{4}$ Division of Diagnostic Pathology, ${ }^{5}$ Department of Pathology, Keio University School of Medicine, Tokyo, Japan. \\ ${ }^{6}$ Department of Cellular Differentiation, Institute for Frontier Medical Sciences, Kyoto University, Kyoto, Japan. \\ 'Department of Biological Information, Tokyo Institute of Technology, Kanagawa, Japan.
}

\begin{abstract}
Valvular heart disease (VHD) is the term given to any disease process involving one or more of the heart valves. The condition can be congenital or acquired, for example as a result of atherosclerosis or rheumatic fever. Despite its clinical importance, the molecular mechanisms underlying VHD remain unknown. We investigated the pathophysiologic role and molecular mechanism of periostin, a protein that plays critical roles in cardiac valve development, in degenerative VHD. Unexpectedly, we found that periostin levels were drastically increased in infiltrated inflammatory cells and myofibroblasts in areas of angiogenesis in human atherosclerotic and rheumatic VHD, whereas periostin was localized to the subendothelial layer in normal valves. The expression patterns of periostin and chondromodulin I, an angioinhibitory factor that maintains cardiac valvular function, were mutually exclusive. In WT mice, a high-fat diet markedly increased aortic valve thickening, annular fibrosis, and MMP-2 and MMP-13 expression levels, concomitant with increased periostin expression; these changes were attenuated in periostin-knockout mice. In vitro and ex vivo studies revealed that periostin promoted tube formation and mobilization of ECs. Furthermore, periostin prominently increased MMP secretion from cultured valvular interstitial cells, ECs, and macrophages in a cell type-specific manner. These findings indicate that, in contrast to chondromodulin I, periostin plays an essential role in the progression of cardiac valve complex degeneration by inducing angiogenesis and MMP production.
\end{abstract}

\section{Introduction}

The prevalence of valvular heart disease (VHD) increases with age, reaching $13 \%$ in individuals 75 years of age or older (1). The morbidity associated with degenerative aortic valve disease is of global interest, given the aging of populations worldwide and the habitual consumption of food high in calories and cholesterol. Several lines of evidence (2-5) suggest that the mechanism of aortic valve degeneration is similar to that of atherosclerosis, namely, infiltration of inflammatory cells and accumulation of oxidized LDL within the valve, proliferation of valvular interstitial cells (VICs), extracellular matrix remodeling, and calcification. Based on this mechanism, prospective, randomized clinical trials of HMG CoA reductase inhibitors for preventing the progression of aortic valve stenosis have been performed, although the results are controversial $(6,7)$. As no preventive pharmacologic therapy for degenerative VHD has been proposed to date, further investigations into the underlying disease mechanisms and the development of novel therapies are warranted.

The previous studies conducted on VHD have largely been observational, immunohistologic, and in vitro studies (8-13). Although the onset of aortic valve stenosis has been reported in Smad6-deficient mice (14), in fibulin 4-deficient mice (15), and

Conflict of interest: The authors have declared that no conflict of interest exists. Citation for this article: JClin Invest. 2010;120(7):2292-2306. doi:10.1172/JCI40973. in humans with the NOTCH1 mutation (16), the abnormality has primarily been observed during cardiac valve development. As avascular tissues, the cardiac valve complex and cartilage share common structural properties $(17,18)$. The cartilage and tendons are known to have unique angioinhibitory mechanisms (19), disruption of which results in angiogenesis and destruction of the joint, leading to arthritis (20). In contrast, angiogenesis and VEGF expression are increased in calcified aortic valves $(21,22)$. Previously, we showed that chondromodulin I (encoded by Lect1) and tenomodulin, angioinhibitory factors expressed in the cartilage and tendon, respectively, were expressed in the cardiac valves and chordae tendineae cordis $(23,24)$, respectively. Whereas chondromodulin I was expressed in the normal cardiac valves, its expression was diminished in areas of angiogenesis in the degenerated valves of human VHD. The absence of chondromodulin I results in angiogenesis and early stage aortic valve stenosis in mice, which indicates that cardiac valve degeneration is promoted by pathologic angiogenesis. The focal absence of tenomodulin is associated with angiogenesis and rupture of the chordae tendineae cordis.

Periostin (encoded by Postn) is a TGF- $\beta$-inducible, $90-\mathrm{kDa}$, secreted protein originally identified in mouse osteoblasts, in which it promotes adhesion and migration $(25,26)$. Periostin is detected as spliced isoforms and contains 4 repeats of the fasciclin domain, which shares homology with the Drosophila protein fasciclin I involved in neuronal cell-cell adhesion (27). In addition to 
the periosteum and periodontal ligament, periostin is expressed in cancer cells, vascular smooth muscle cells, fibroblasts, and wound-site blood vessels and participates in tumor angiogenesis, metastasis, and cell migration (28-31). In the heart, periostin is physiologically expressed in embryonic cardiac valves, while it is reexpressed abundantly in adult LV after pressure overload or myocardial infarction (32-37). Although heart size and cardiomyocyte number are unchanged at baseline in Postn ${ }^{-/-}$mice, LV remodeling and hypertrophy are attenuated without apparently affecting the proliferation of cardiomyocytes and cardiac fibroblasts, which suggests crucial effects of periostin on LV fibrosis and hypertrophy after cardiac insult $(34,35)$. Previous studies have clearly demonstrated the physiologic role of periostin in the cardiac valve and its critical involvement in cardiac valve maturation during development $(38,39)$. However, it remains unknown whether periostin plays any pathophysiologic role in adult valvular function.

During our investigation of degenerated human cardiac valves, we unexpectedly found that periostin was strikingly increased in patients with atherosclerotic and rheumatic VHD. This finding led us to hypothesize that periostin plays a distinct pathophysiologic role in degenerated cardiac valves. The present study demonstrates, for the first time to our knowledge, the involvement of periostin in the process of cardiac valve complex degeneration using human surgical specimens. We also investigated the pathophysiologic role of periostin in VHD using high-fat (HF) diet-induced degeneration of the cardiac valve complex and its rescue in Postn ${ }^{-/-}$mice. We further clarified the molecular mechanism by analyzing the in vitro effects of periostin on ECs, VICs, and engrafted macrophages.

\section{Results}

Periostin isoforms are specifically expressed in the cardiac valves and annuli of rodent hearts from the embryonic stage to adulthood. Because murine periostin is known to have 4 spliced isoforms within the C-terminal domain (Figure 1A, right), we initially determined the temporal and spatial expression patterns of the periostin isoforms in embryonic and adult mouse hearts. The RT-PCR analysis revealed that the periostin isoforms were first expressed in the E8.5 heart, and their levels increased thereafter (Figure 1A, left). Interestingly, the shift from the long isoform to the short isoform of periostin mRNA occurred in the postnatal heart. Western blot analysis revealed that the relative expression level of this protein in a heart decreased postnatally, probably as a result of its limited expression in valvular areas (Figure 1B). Furthermore, the short isoform was predominantly expressed in adult murine aortic valves, whereas the long isoform was mainly expressed in the mitral valves (Figure 1C).

Immunofluorescence staining with an anti-periostin antibody that recognizes both long and short isoforms showed that periostin was specifically expressed in the outflow tract and atrioventricular canal at E11.5 and in the cardiac valves and their annuli thereafter (Figure 1D). At 4 postnatal weeks, periostin expression appeared to be more localized to the subendothelial superficial layers of the cardiac valves and annuli.

Immunohistochemistry (IHC) of the adult rat cardiac valves confirmed that periostin was expressed in all 4 (aortic, mitral, pulmonary, and tricuspid) valves and in their annuli, in which the expression pattern of periostin was similar to that seen in murine valves (Figure 1E). These results indicate that periostin is expressed from E8.5 to adulthood in all 4 cardiac valves and that its expression is localized to the subendothelial superficial layers of the cardiac valves in adult rodent hearts.
Physiologic expression of periostin is localized primarily to the zona ventricularis/atrialis and zona fibrosa in adult human cardiac valves. Next, we investigated periostin expression in adult human normal cardiac valves obtained at autopsy. IHC revealed that periostin was expressed in the superficial layers of the normal aortic and mitral valves, whereas it was expressed throughout the murine embryonic valves (Figure 2, A and B, and Supplemental Figure 1; supplemental material available online with this article; doi:10.1172/JCI40973DS1). Of note, periostin was strongly expressed at the zona ventricularis side of the aortic valve and the atrialis side of the mitral valve, and to a lesser extent at the zona fibrosa of both, just beneath the ECs.

We also performed triple immunofluorescence staining for periostin and other components of the aortic valves. Interestingly, periostin was expressed in the subendothelial superficial layer, whereas chondromodulin I, an angioinhibitory protective factor in the cardiac valve (23), was expressed in the core layer of the cardiac valve (Figure 2C). Therefore, the expression patterns of these 2 proteins are mutually exclusive. The expression pattern of periostin did not coincide with that of either vWF or collagen I (Figure 2, $\mathrm{D}$ and $\mathrm{E}$ ). Human cardiac valves consist of 3 distinct layers of extracellular matrix (fibrosa, spongiosa, and atrialis-ventricularis), and elastin is the predominant component of the atrialis and ventricularis (40). Indeed, the regions of periostin expression largely overlapped those of elastin (Figure 2F). Similar expression patterns were observed in the mitral valves (Supplemental Figure 2).

Periostin expression is strikingly upregulated and expanded in patients with atherosclerotic and rheumatic VHD. To investigate whether periostin is involved in the pathogenesis of VHD, we compared its expression profiles in normal human cardiac valves and in the cardiac valves of patients with VHD. Pathologic cardiac valves obtained by valvular replacement surgery, including atherosclerotic, rheumatic, and prolapse valves ( $n=8,9$, and 4 , respectively), were examined by IHC. Unexpectedly, we found not only that periostin expression was markedly elevated in the subendothelial superficial layer, but also that the area of periostin expression was expanded in the cardiac valves in patients with degenerative VHD, such as those with atherosclerotic or rheumatic valves. In the periostin-positive areas of the atherosclerotic valves, chondromodulin I expression was absent, whereas VEGF was upregulated and the small vessels were heavily infiltrated (Figure 3A). Similar findings were observed for rheumatic valves, whereas the periostin expression pattern did not show any significant changes in patients with mitral valve prolapse (Supplemental Figure 3).

Quantitative analysis revealed that the percentage of the expression area of periostin was increased 4.0-fold in patients with atherosclerotic and rheumatic valves, but not in those with valvular prolapse (Figure 3B). In patients with atherosclerotic or rheumatic valves, the chondromodulin I-positive areas were decreased to $25 \%$, and the small vessel density, detected by vWF staining and VEGF expression, was markedly elevated, as we reported previously (23).

Western blot analysis revealed that the levels of periostin expression increased 6.1-fold and 3.2-fold in atherosclerotic and rheumatic VHD, respectively (Figure 3C). Concomitant with these increases in periostin, the levels of expression of $\alpha$-SMA and collagen I were drastically increased in these patients.

Next, we examined the relationships between the areas of angiogenesis and periostin expression in these forms of VHD. IHC showed that neoangiogenesis occurred mainly at the zona atrialis/ ventricularis and zona spongiosa in the mid-regions of the valves, especially in areas in which normal vWF expression was dimin- 

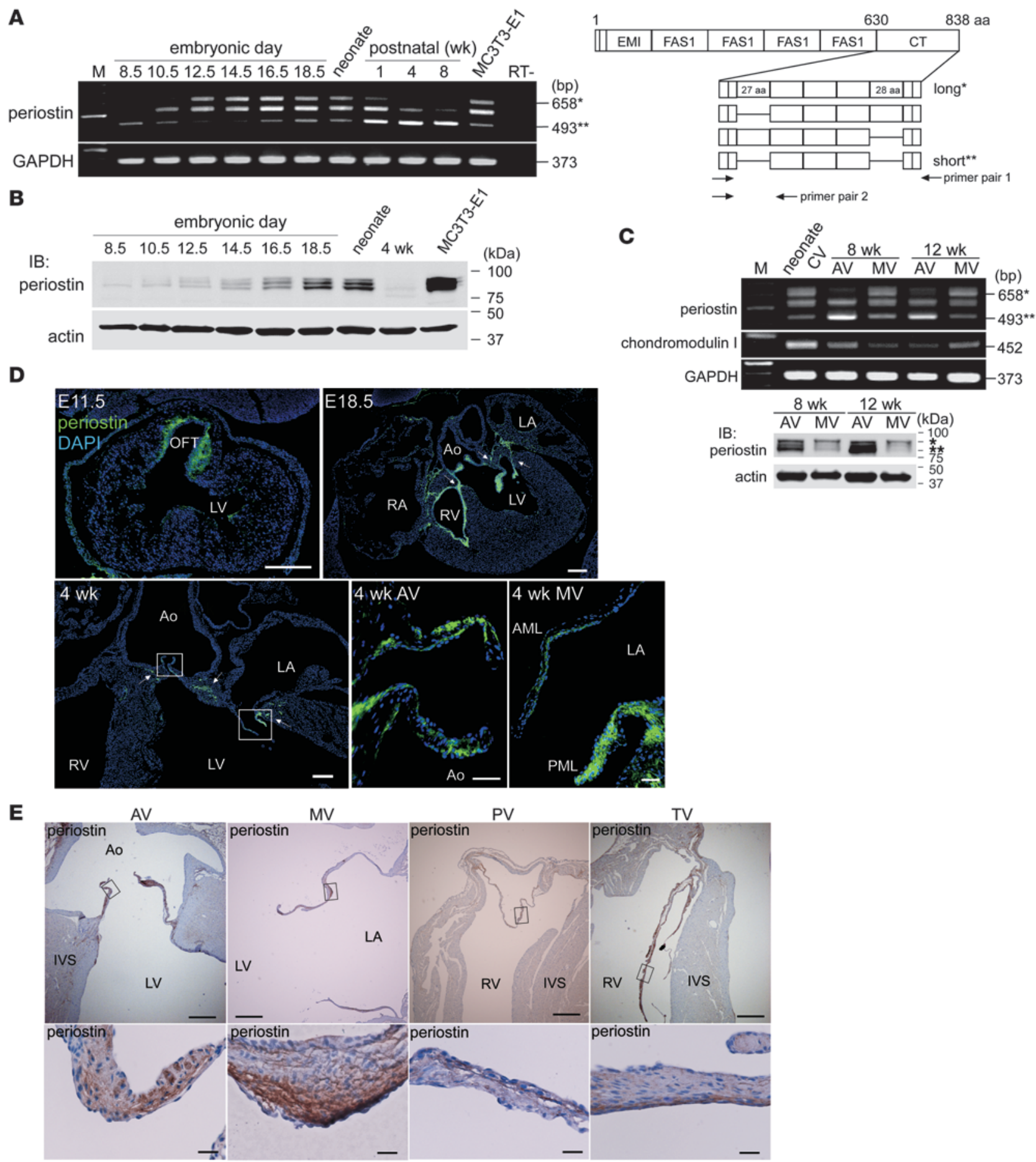

Figure 1

Periostin isoforms are specifically expressed in the cardiac valves and annuli of embryonic and adult rodent hearts. (A and B) RT-PCR (A) and Western blot (B) analyses of periostin isoforms in the mouse heart. The primer pair 1 for murine periostin (A, right) was used for PCR. Each box in the C-terminal (CT) domain represents an exon. Single and double asterisks represent the long and short periostin isoforms, respectively. Periostin was first detected in the hearts at E8.5, and the relative expression level of this protein decreased postnatally. MC3T3-E1 cells are a positive control. M, marker; FAS1, fasciclin I domain. (C) RT-PCR (top) and Western blot (bottom) analyses of the periostin isoforms in adult mouse cardiac valves (CV). AV, aortic valve; MV, mitral valve. (D and E) Immunostaining for periostin in the hearts of mice at E11.5 to 4 weeks of age (D) and in the heart of a 6-week-old rat (E). In D, periostin was specifically expressed in the outflow tract (OFT), cardiac valves, and annuli (arrows). In E, periostin (brown) was expressed in all 4 cardiac valves and their annuli. The boxed regions are shown at higher magnification. Note that periostin was localized to the subendothelial superficial layer of the adult cardiac valve. AML, anterior mitral leaflet; Ao, aorta; IVS, interventricular septum; LA, left atrium; PML, posterior mitral leaflet; PV, pulmonary valve; RA, right atrium; TV, tricuspid valve. Scale bars: 200 um (D); $50 \mu \mathrm{m}$ (D, higher magnification); $500 \mu \mathrm{m}$ (E); $20 \mu \mathrm{m}$ (E, higher magnification). 


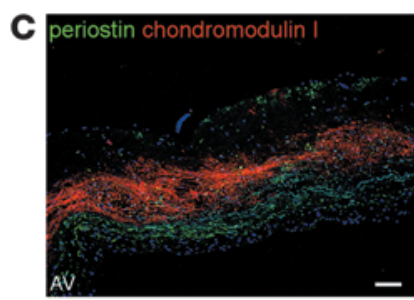

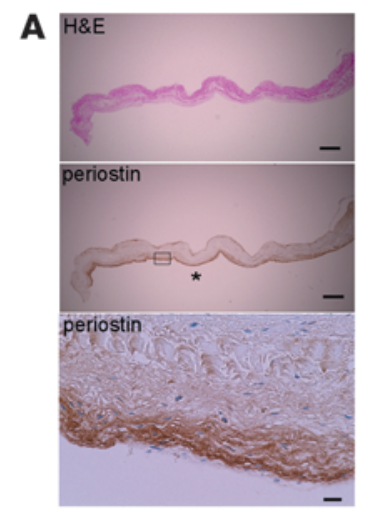
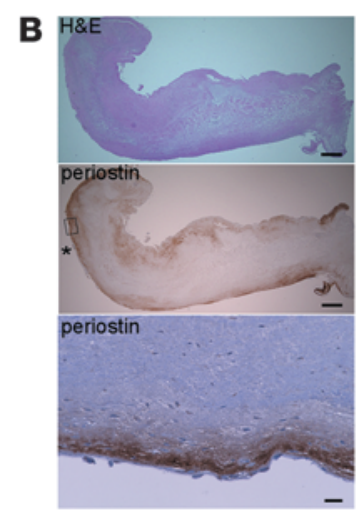

E

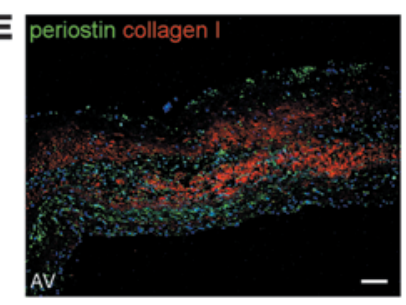

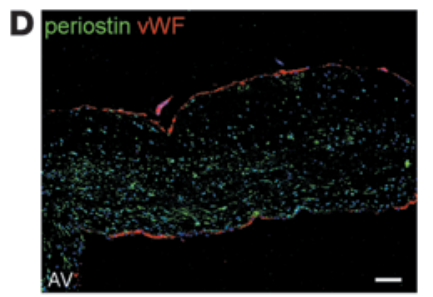

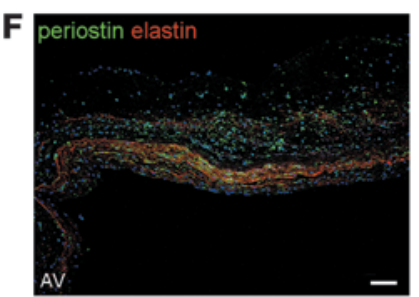

ished in the valve endocardium (Supplemental Figure 4A). We also found that periostin expression was specifically increased in the areas of neoangiogenesis in the degenerated valves (Supplemental Figure 4B). To identify the cell types that synthesize periostin in VHD, double immunofluorescence staining with periostin and $v W F, \alpha$-SMA, vimentin, or the activated monocyte/macrophage marker CD14 was performed. Immunofluorescence staining revealed that periostin expression was increased in the interstitial tissues of the areas of angiogenesis, into which inflammatory cells and myofibroblasts had infiltrated (Figure 4A). Indeed, periostin was coexpressed with $\alpha$-SMA, vimentin, and CD14 in these cells (Figure 4B). Immunofluorescence staining and Western blot analysis further confirmed that periostin was expressed and secreted from CD14-positive, cultured mouse BM-derived macrophages (Figure 4, C and D). These results indicate that periostin expression in degenerative atherosclerotic and rheumatic VHD, but not in prolapsed VHD, is completely different from its physiologic expression. In these forms of VHD, levels of periostin are markedly increased in the interstitial tissues of the newly formed small vessel areas, and periostin is secreted from the infiltrated inflammatory cells and myofibroblasts.

HF diet-induced thickening of the aortic valves and annuli is attenuated in Postn ${ }^{-/-}$mice. Our initial results led us to investigate whether the increased expression of periostin plays an essential role in cardiac valve degeneration or is merely an epiphenomenon. To resolve this issue, we generated Postn ${ }^{-/}$mice of C57BL/6 strain (Figure 5A), and WT and Postn ${ }^{-/-}$mice at 12 weeks of age were fed either normal or HF diet for 4 months; C57BL/ 6 mice are the most atherosclerosis sensitive among mouse strains (41), and the HF regimen is

\section{Figure 2}

Physiologic expression of periostin is localized primarily to the zona ventricularis/atrialis and zona fibrosa in adult human cardiac valves. Representative, consecutive sections of normal human cardiac valves subjected to IHC (A and $\mathbf{B}$ ) and triple-immunofluorescence staining (C-F). (A and $\mathbf{B}$ ) IHC sections of the aortic valves $(\mathbf{A})$ and mitral valves $(\mathbf{B})$. The boxed regions in the periostin-stained sections are shown at higher magnification below. Prominent expression of periostin was observed at the ventricularis and atrialis sides (asterisks) and to a lesser extent at the zona fibrosa, just beneath the ECs. (C-F) Localization of periostin (green) and other components (red) in the normal aortic valves. Nuclei are stained blue. Since periostin was expressed in the subendothelial superficial layer and chondromodulin I in the core layer of the valve, the expression patterns of these proteins are mutually exclusive (C). The expression pattern of periostin overlapped with that of elastin $(\mathbf{F})$, but not those of vWF and collagen I (D and E). Scale bars: $500 \mu \mathrm{m}$ (A and B); $20 \mu \mathrm{m}$ (A and B, higher magnification); $100 \mu \mathrm{m}$ (C-F).

known to cause significant thickening of their aortic valves, resulting in early-stage aortic valve stenosis (42). We found that the HF diet caused similar levels of obesity and hypercholesterolemia in WT and Postn ${ }^{-1-}$ mice (Figure 5B).

We next performed 45-MHz echocardiography, which revealed that the HF diet produced high-echogenic areas in the aortic and mitral valve annuli in the HF diet-fed WT mice (Figure 5C). Moreover, the M-mode of echocardiography demonstrated that the aortic valve was apparently thickened in WT mice fed the HF diet compared with the normal diet. Surprisingly, the high-echogenic areas and aortic valve thickening were strongly attenuated in the HF diet-fed Postn ${ }^{-/}$mice. Using quantitative analyses, we confirmed that the aortic valve thicknesses and echogenic areas of the annuli were significantly increased in HF diet-fed WT mice compared with WT mice fed normal diet, whereas these changes were strongly reduced in the HF diet-fed Postn ${ }^{-/}$mice (Figure 5D). The wall thickness, internal diameter, and ejection fraction of the LV were unchanged in all the groups (Supplemental Figure 5). These results suggest that periostin is intrinsically involved in $\mathrm{HF}$ diet-induced degeneration of the cardiac valve complex.

$H F$ diet-induced fibrosis and MMP expression in the cardiac valve complex are reduced in Postn ${ }^{-1-}$ mice. To investigate further the role of periostin in valve degeneration, IHC and Western blot analysis were performed. Through quantitative analysis of the IHC sections, we confirmed that the HF diet increased the areas of periostin expression 2.2- to 2.5-fold in the aortic and mitral valve complexes of the WT mice (Figure 6, A and B). In addition, the areas of expression of VWF, collagen I, and $\alpha$-SMA were increased in the HF diet-fed WT mice compared with WT mice fed normal diet, whereas expression levels of these proteins were markedly reduced in $\mathrm{HF}$ diet-fed Postn ${ }^{-/-}$mice.

The mitral valve complexes were then excised from the mice and subjected to Western blot analysis. The expression levels of periostin, collagen I, and $\alpha$-SMA were significantly upregulated in the HF diet-fed WT mice and decreased in the HF diet-fed Postn $n^{-1-}$ mice, confirming the IHC results (Figure 6C). It is well known that MMPs play critical roles in tissue remodeling and angiogenesis and are closely linked to the progression of atherosclerosis, aortic aneurysm, LV remodeling, and arthritis (43). Among the MMPs, MMP-13 is considered to fulfill the role of MMP-1 in rodents, which do not express the latter protein postnatally (44). Moreover, it has been reported previously that the levels of MMP-1, MMP-2, MMP-9, and MMP-13 are increased in the car- 
A
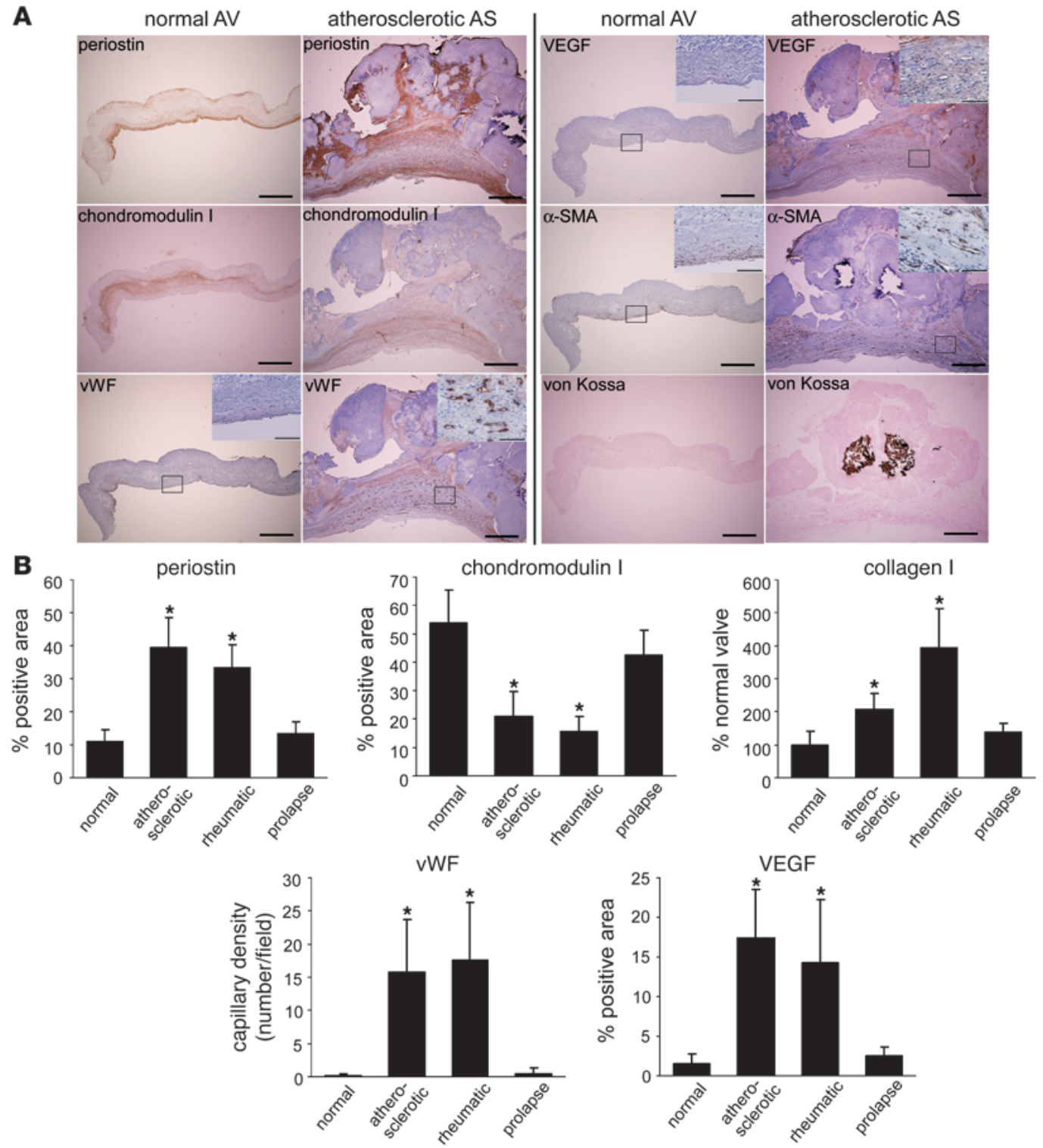

C
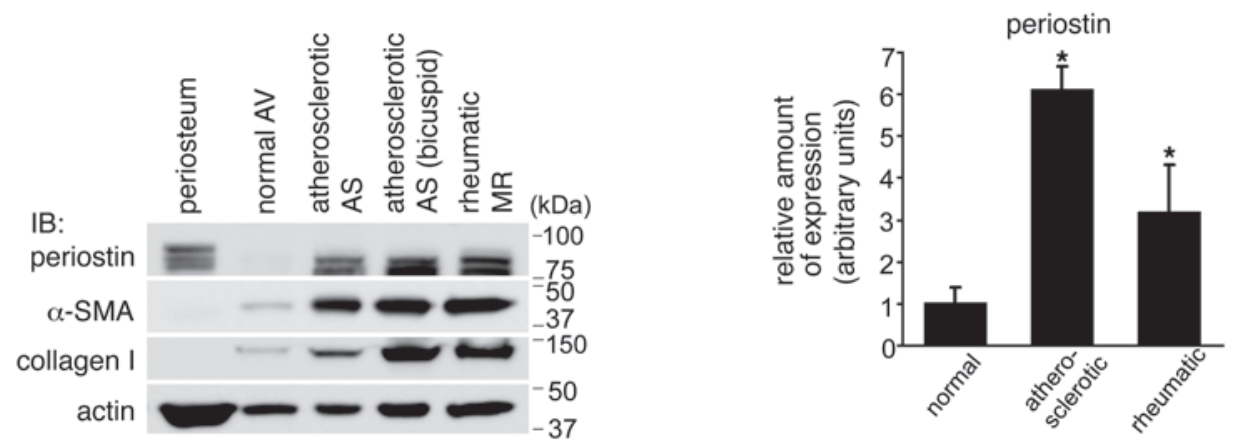

Figure 3

Periostin expression is strikingly upregulated and expanded in patients with atherosclerotic and rheumatic VHD. (A) Representative, consecutive IHC sections of normal aortic valves and atherosclerotic aortic valve stenosis. Boxed regions are shown at higher magnification in the insets. (B and C) Quantitative analyses of the expression of periostin, chondromodulin I, vWF, VEGF, $\alpha$-SMA, and collagen I in normal valves and VHD valves, as measured by IHC (B) and Western blotting (C). The areas of expression of periostin, vWF, VEGF, $\alpha$-SMA, and collagen I were strikingly expanded, whereas that of chondromodulin I was significantly reduced, in the atherosclerotic and rheumatic valves compared with the normal valves. The level of periostin expression was markedly increased in the atherosclerotic valves, and to a lesser extent in the rheumatic valves, as assessed by densitometric analysis of the Western blot. AS, aortic valve stenosis; MR, mitral valve regurgitation. Scale bars: $500 \mu \mathrm{m}$; $100 \mu \mathrm{m}$ (insets). ${ }^{*} P<0.05$ versus normal. 
A

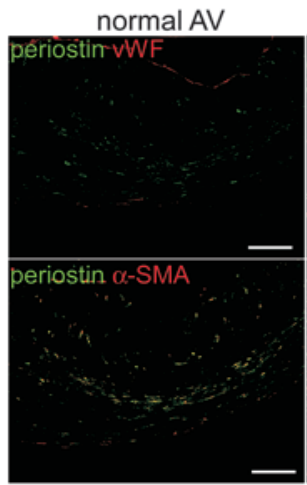

B

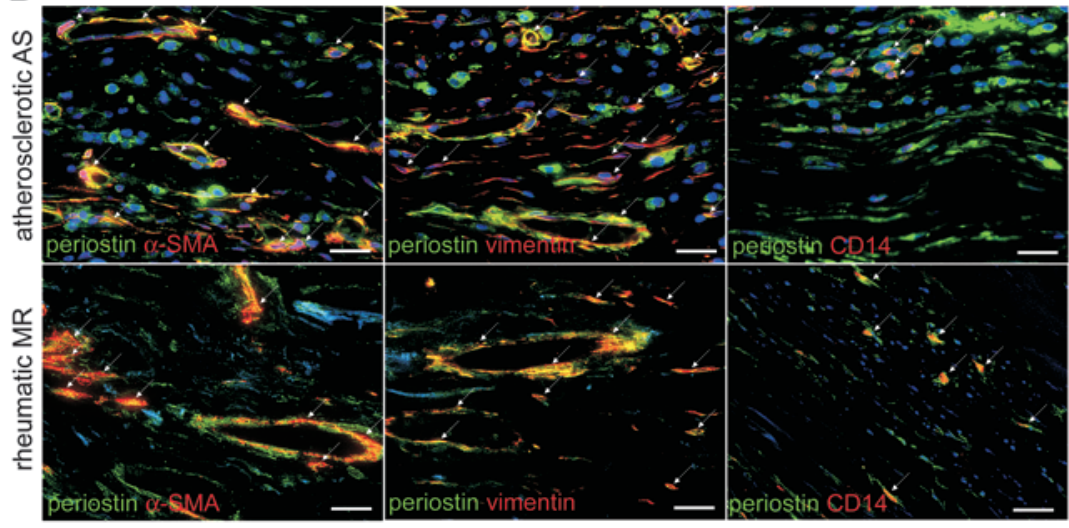

atherosclerotic AS
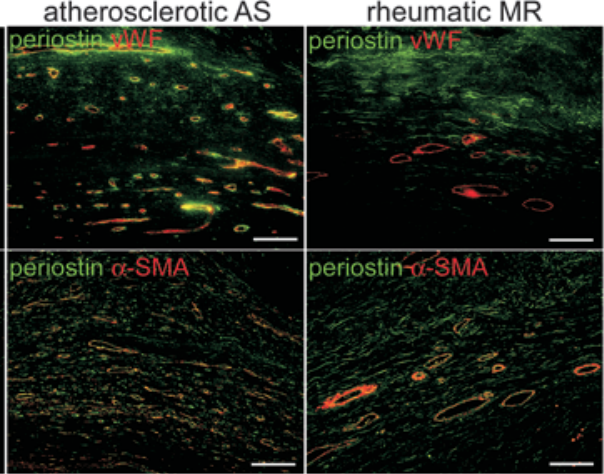

D
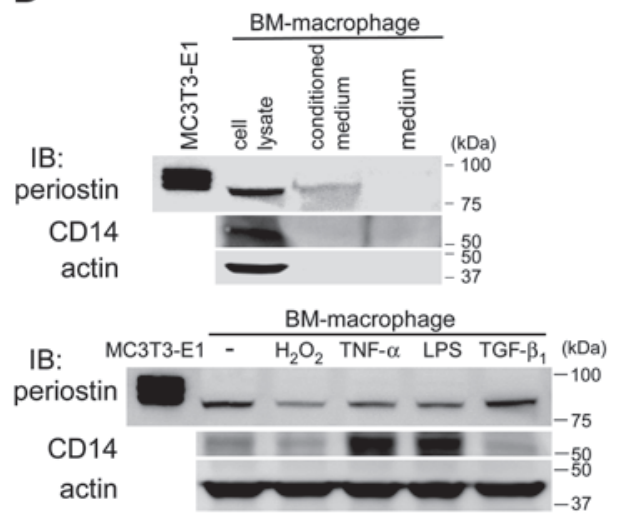

Figure 4

Periostin expression is specifically increased in the interstitial tissues of the neoangiogenesis areas in human VHD. (A and B) Tripleimmunofluorescence staining for periostin (green) and vWF, $\alpha$-SMA, vimentin, or the activated monocyte/macrophage marker CD14 (red). Nuclei are stained blue in B. Note that periostin was coexpressed with $\alpha$-SMA, vimentin, and CD14 (arrows). (C and D) Double-immunofluorescence staining (C) and Western blot (D) analyses of periostin in cultured mouse BM-derived macrophages. MC3T3-E1 was used as a positive control for periostin expression. Periostin expression levels in macrophages treated with various stimuli for 24 hours are shown at the bottom of $\mathbf{D}$. Periostin was expressed and secreted by the CD14-positive macrophages. Scale bars: $100 \mu \mathrm{m}(\mathbf{A}) ; 20 \mu \mathrm{m}$ (B and C).

diac valves of patients with VHD $(23,45-47)$. We found that the expression levels of MMP-2 and MMP-13 significantly increased in HF diet-fed WT mice, whereas this increase was strongly reduced in HF diet-fed Postn ${ }^{-1-}$ mice (Figure 6C). We also found modest calcification of the mitral valve annuli in HF diet-fed WT mice, but not in HF diet-fed Postn ${ }^{-/-}$mice (Supplemental Figure 6). We considered that the increase in the echogenic area of the annuli of the HF diet-fed WT mice may be caused by enhanced fibrosis and calcification. These results demonstrate that periostin mediates the HF diet-induced increases in fibrosis and MMP expression in the cardiac valve complex.

Periostin promotes angiogenesis in ECs both in vitro and ex vivo. To analyze further the function of periostin in cardiac valve degeneration, we performed in vitro experiments using ECs. As the differential roles of the periostin isoforms remained to be clarified, we collected the conditioned media of cultures of COS7 cells that expressed the long or short isoform of murine periostin as a result of being transfected with recombinant adenoviruses (Figure 7A), and the effects of these conditioned media on EC functions were examined. Previous studies have shown that periostin is expressed in aortic ECs, but not in valvular ECs, and that mesenchyme-specific cadherin 11 is expressed in valvular ECs, but not in aortic ECs, indicating phenotypic differences between valvular endocardial ECs and arterial ECs $(48,49)$. Using immunofluorescence staining and Western blotting, we found that cadherin 11, but not periostin, was expressed in human coronary artery ECs as well as in the ECs located in areas of neoangiogenesis in the stenotic aortic valves (Supplemental Figure 7). In contrast, periostin, but not cadherin 11, was expressed in aortic ECs. Therefore, human coronary artery ECs, rather than aortic ECs, were used in the in vitro angiogenesis assay.

Both the long and short periostin isoforms significantly and comparably promoted tube formation, migration, 3D lumen formation, and focal adhesion kinase (FAK) activation in ECs (Figure 7, B-E), in agreement with a previous report (50). Furthermore, periostin partially decreased the number of annexin $\mathrm{V}$-positive apoptotic ECs induced by serum starvation and activated Akt without affecting EC proliferation (Figure 7, F-H, and Supplemental Figure 8).

The ex vivo aortic ring assay revealed that neoangiogenesis outward from the aortic roots and annuli in Postn ${ }^{-/-}$mice was strikingly inhibited compared with that in WT mice, and that periostin supplementation partially rescued this deficit (Supplemental Figure 9). Taken together, these results indicate that periostin itself promotes angiogenesis in ECs both in vitro and ex vivo and that the effects of the 2 periostin isoforms are comparable.

Periostin induces secretion of MMPs from VICs, ECs, and macrophages in vitro. Next, we investigated the effect of periostin on the functions of VICs, the predominant cell type in the cardiac valve. Cultured rat VICs, which stained positively for chondromodulin I and nega- 
research article

A
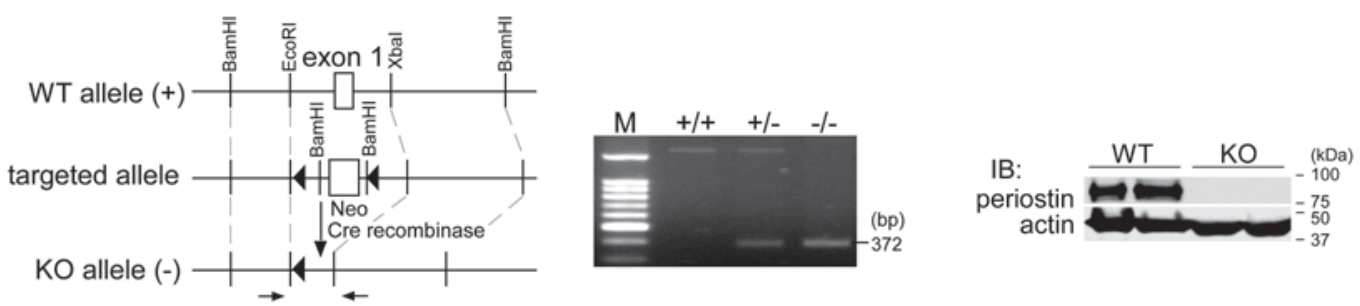

B
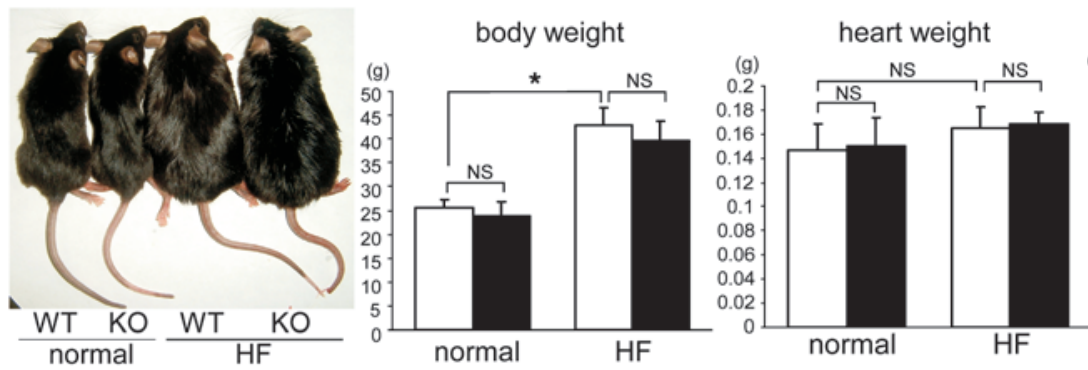

serum total cholesterol

C
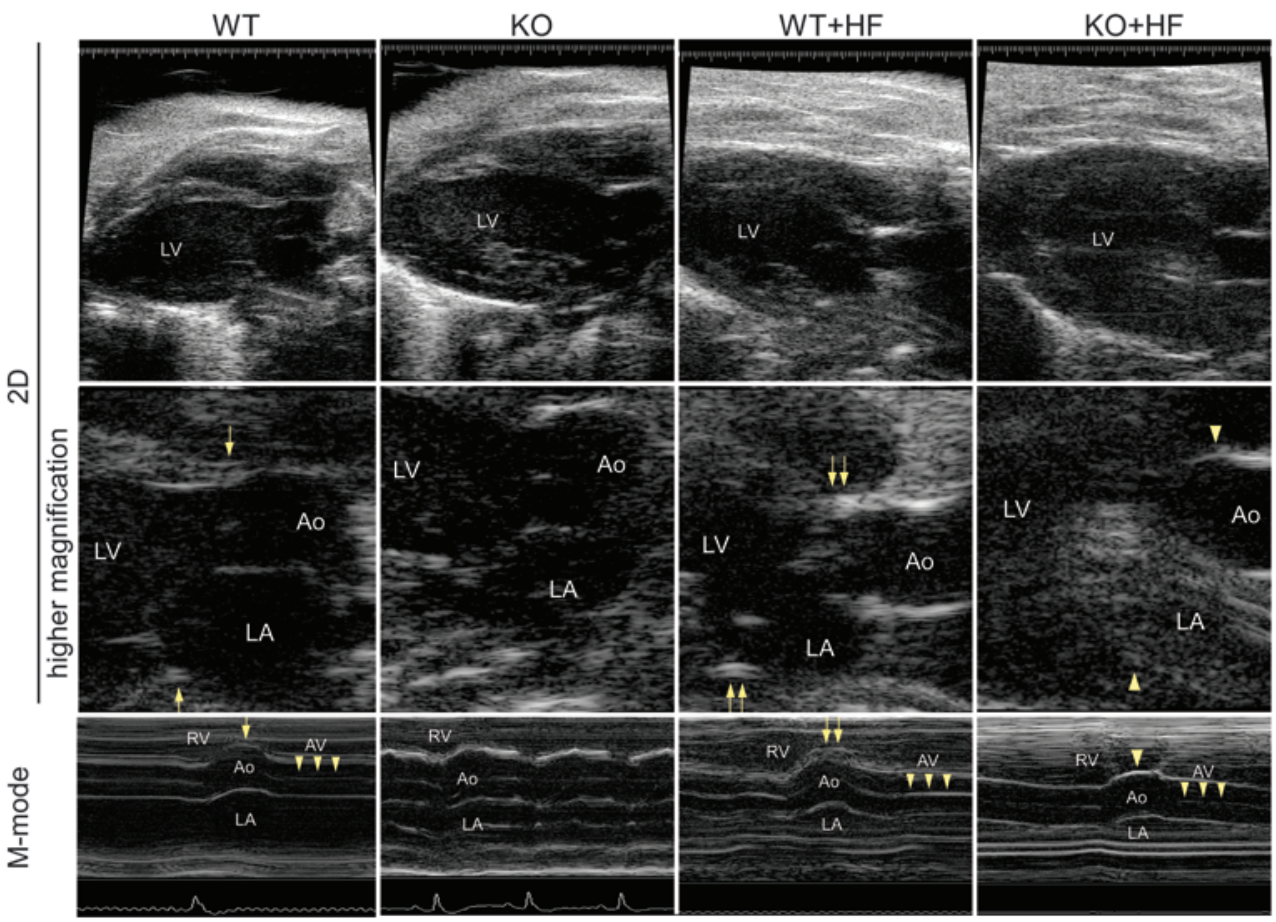

D
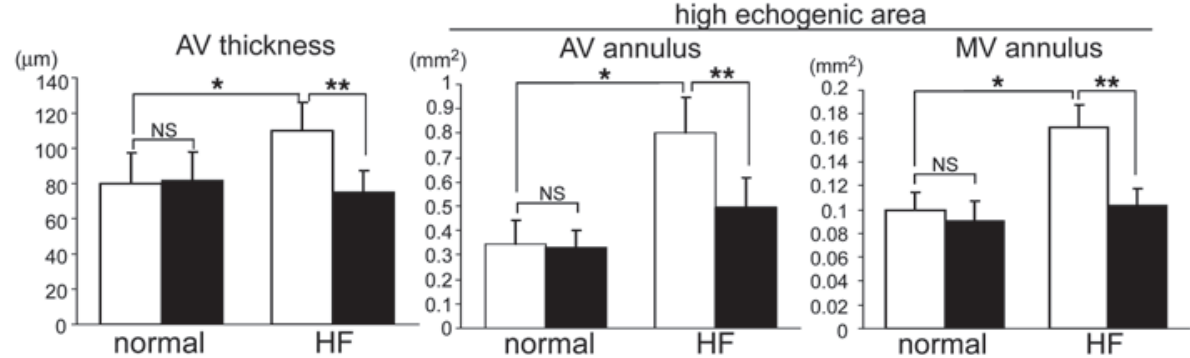

$\square \mathrm{WT}$ 


\section{Figure 5}

HF diet-induced thickening of the aortic valves and annuli is attenuated in Postn ${ }^{-/}$mice. (A) Generation of Postn ${ }^{-/}$mice. Schema of the strategy used for targeting the first exon of the murine Postn gene. Genotyping of mouse offspring was performed by PCR of tail-DNA samples. The primer pair used is indicated by arrows in the schema. Also shown is Western blot analysis of the periostea in the WT and Postn-1- (KO) mice. (B) Appearance of mice fed normal diet or HF diet for 4 months. The HF diet caused similar levels of obesity and hypercholesterolemia in both WT and Postn ${ }^{-/}$mice. (C and D) Representative images (C) and quantitative analyses of the aortic valve thicknesses and echogenic areas of the annuli (D), as assessed by $45-\mathrm{MHz}$ echocardiography. In C, images were taken at the diastolic phase using the 2D mode, and at the level of the aortic valve using the M-mode of the left parasternal long axis view. The echogenic areas of the aortic and mitral valve annuli greatly increased in the HF diet-fed WT mice (double arrows) compared with those in the WT mice (arrows). These changes were reduced in the HF diet-fed Postn $^{-1-}$ mice (arrowheads). Furthermore, the HF diet-induced thickening of the aortic valves was significantly attenuated in the HF diet-fed Postn ${ }^{-1}$ mice (triple arrowheads). Original magnification, $\times 10 ; \times 40$ (higher magnification). ${ }^{\star} P<0.05$ versus WT; ${ }^{* *} P<0.05$ versus HF diet-fed Postn ${ }^{-1}$.

tively for acetylated LDL (Figure 8A), were prepared as described previously (23). Periostin neither affected VIC proliferation (Supplemental Figure 10A) nor reduced serum starvation-induced apoptosis (Supplemental Figure 10B). Interestingly, however, Western blot analysis revealed that periostin strikingly increased the secretion of MMP-2 and MMP-13 from VICs, whereas it did not affect MMP-9 secretion (Figure 8B). RT-PCR analysis showed that periostin strongly induced the transcription of $M m p 13$, but not that of Mmp2 (Figure 8C). Moreover, it significantly stimulated the secretion of MMP-2 and MMP-9 from ECs and cultured BMderived macrophages, respectively (Figure 8, D and E), suggestive of cell type-specific induction of MMPs by periostin. These results demonstrate that periostin promotes the secretion of MMPs from VICs, ECs, and macrophages in vitro. Our in vitro findings strongly support the notion that periostin plays a critical role in promoting cardiac valve complex degeneration.

Periostin and chondromodulin I do not cross-regulate each other in the cardiac valves. The reciprocal expression patterns and functions of periostin and chondromodulin I led us to consider whether these proteins cross-regulate each other in the cardiac valves. To examine this possibility, the cardiac valves of Postn ${ }^{-/}$and Lect $1^{-/-}$mice (23) were examined for chondromodulin I and periostin expression, respectively, using IHC and Western blot analysis. We found that chondromodulin I expression was not altered in the valves of HF diet-fed WT and Postn ${ }^{-/-}$mice (Supplemental Figure 11A). Conversely, periostin expression was unchanged in the valves and annuli of the WT and Lect1 $1^{-1-}$ mice (Supplemental Figure 11, B and C). Moreover, periostin stimulation affected neither the transcription nor the translation of chondromodulin I in rat VICs in vitro (Supplemental Figure 11D). These results indicate that periostin and chondromodulin I do not cross-regulate each other in the cardiac valves.

\section{Discussion}

Previous studies showed that periostin plays an important role in cardiac valve development $(38,39)$. However, the results of the present study demonstrate that periostin has a distinct role in the progression of cardiac valve degeneration in atherosclerotic and rheumatic VHD. Periostin expression was localized to the suben- dothelial superficial layer of normal cardiac valves and annuli in adult human hearts, while it was greatly increased in the areas of neoangiogenesis in the degenerated valves of patients with VHD. Moreover, we also found that HF diet induced degeneration of the cardiac valve complex in WT mice, whereas this degeneration was markedly rescued in Postn ${ }^{-/-}$mice via the prevention of fibrosis and MMP expression. Finally, we found that periostin affected the functions of major cellular components of the degenerated valves, in that it not only promoted angiogenesis in ECs, but also increased the secretion of MMPs from VICs, ECs, and engrafted macrophages in vitro. The reciprocal expression patterns and functions of periostin and chondromodulin I clearly define the degenerative role of periostin and the protective role of chondromodulin I in the progression of degenerative VHD.

A recent study reported that periostin expression was decreased in the valves of infants with congenital bicuspid aortic valve stenosis (39). Moreover, the authors revealed that Postn ${ }^{-/-}$mice exhibited truncated and disorganized cardiac valves. That study showed that decreased physiologic expression of periostin during the developmental stage resulted in congenital valve deformities. In contrast, we focused for the first time to our knowledge on the expression and role of periostin in adult-acquired VHD, particularly in degenerative atherosclerotic and rheumatic VHD. We found that periostin was pathologically overexpressed in these forms of VHD, in which it was abundantly secreted by infiltrated inflammatory cells and myofibroblasts. It is possible that these myofibroblasts are recruited from circulating fibrocytes, as it has previously been shown that bone marrow-derived hematopoietic stem cells contribute to the adult cardiac VIC population (51) and that peripheral blood fibrocytes originating from hematopoietic stem cells or CD14-positive peripheral blood monocytes can differentiate into myofibroblasts in several tissues (52). In these instances, the origins of the periostin-producing cells were completely dissimilar to those of the cells involved in its physiologic expression. In contrast, the expression levels of periostin were significantly lower, and those of collagen I were significantly higher, in the valves of rheumatic VHD than in those of atherosclerotic VHD. Given these findings, it might be appropriate to describe rheumatic VHD as a phenotype of accelerated formation and reorganization of extracellular matrices rather than as a phenotype of degeneration.

We also demonstrated that periostin had tube-forming and migratory activities in ECs both in vitro and ex vivo, and we confirmed that these activities were mediated by the activation of FAK and Akt, which indicated that periostin has angiogenic activity. It is well known that the normal cardiac valve is an avascular tissue, whereas the degenerated valve contains numerous small vessel infiltrations. We believe that periostin upregulation in the degenerated valves is critical for small vessel formation and for collaboration with other angiogenic factors (53). In support of our observations, the angiogenic activity of periostin has been implicated in tumor angiogenesis $(29,50,54)$. Although the precise periostin-mediated angiogenic mechanisms within the valves are not elucidated in the present study, we demonstrated that neoangiogenesis occurred mainly in the zona atrialis/ventricularis and spongiosa in the midregions of the valves, particularly at sites in which the normal structure of the valve endocardium is disrupted. Therefore, periostin may enhance the recruitment of circulating endothelial progenitor cells by promoting their adhesion and migration into the valves, or it might enhance the penetration of the microvessels from the annulus region or roots into the leaflets. 
A

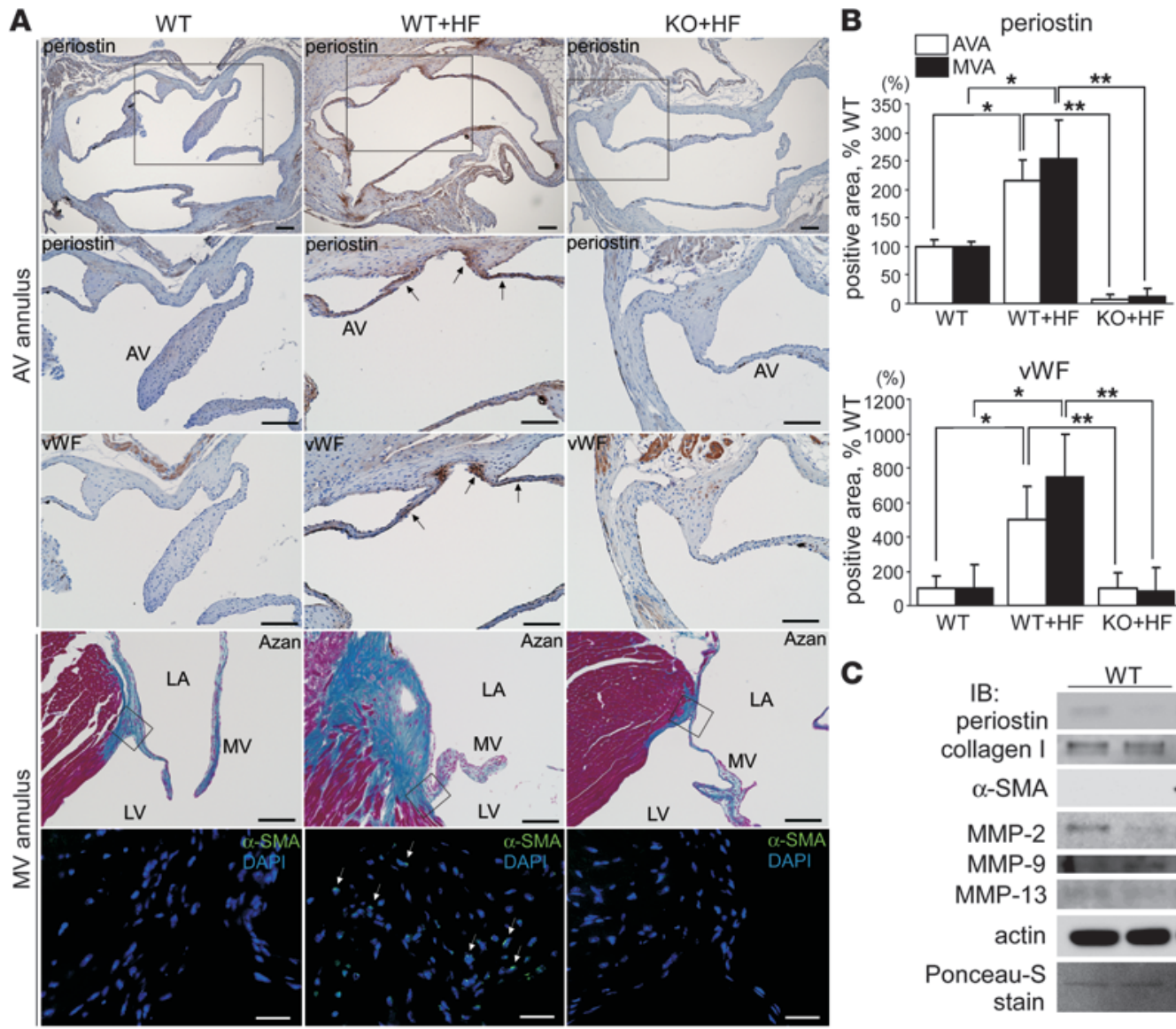

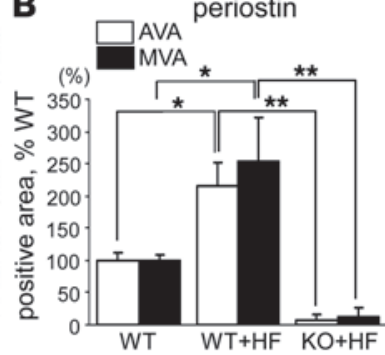
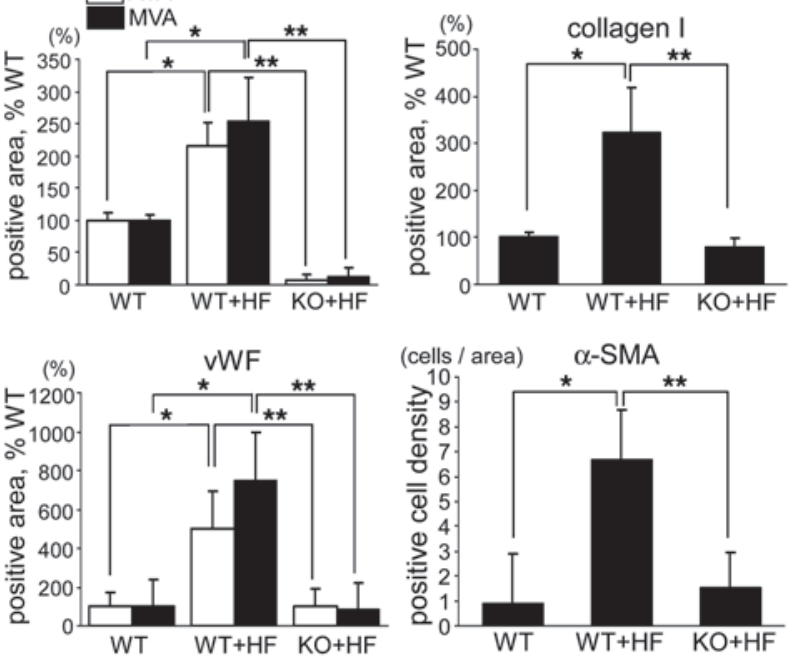

C

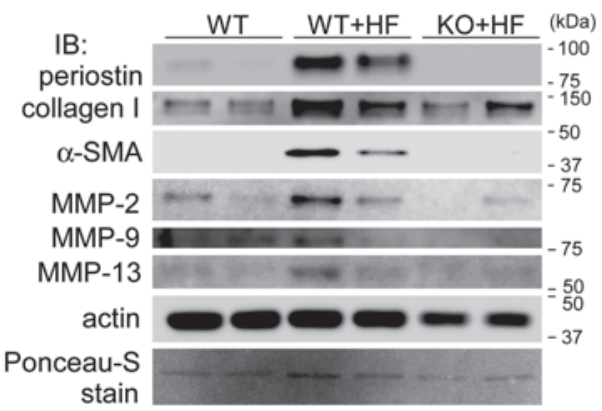

Figure 6

HF diet-induced fibrosis and MMP expression in the cardiac valve complex are reduced in Postn ${ }^{-/-}$mice. (A-C) Representative IHC sections (A), quantitative analyses of the expression areas (B), and Western blot analysis (C) of the cardiac valve complexes in WT and Postn ${ }^{-1-}$ mice. (A) Horizontal sections of AV annuli and longitudinal sections of MV annuli are shown. The boxed regions in the periostin-stained sections are shown at higher magnification immediately below, and the boxed regions in the Azan-stained sections are shown as $\alpha$-SMA immunofluorescence below. The areas of expression of periostin, vWF, and $\alpha$-SMA and the Azan-stained areas were markedly expanded in the HF diet-fed WT mice (arrows), whereas these changes were reduced in the HF diet-fed Postn ${ }^{-/}$mice. (B) The areas of expression in the aortic valve annulus (AVA) and mitral valve annulus (MVA) are shown as percentages relative to WT mice. (C) Murine mitral valves with annuli were excised and subjected to Western blot analysis. The expression levels of periostin, collagen I, $\alpha$-SMA, MMP-2, and MMP-13 were greatly increased in the HF diet-fed WT mice and reduced in HF diet-fed Postn ${ }^{-/}$mice. Scale bars: $100 \mu \mathrm{m} ; 20 \mu \mathrm{m}\left(\alpha-S M A\right.$ immunofluorescence). ${ }^{*} P<0.05$ versus WT; ${ }^{* \star} P<0.05$ versus HF diet-fed $P$ ostn ${ }^{-1}$.

MMPs are critically involved not only in appropriate embryonic development, but also in the progression of various degenerative diseases. Previously, we reported that MMP-1, MMP-2, MMP-9, and MMP-13 were overexpressed in areas of neoangiogenesis in patients with degenerative VHD, although the precise mechanisms were unknown (23). The present study clearly shows that periostin increased secretion of MMP-2, MMP-9, and MMP-13 from VICs, ECs, and cultured macrophages in a cell type-specific manner. Moreover, periostin upregulated MMP-2 expression in VICs by posttranscriptional mechanisms, possibly by inhibiting MMP-2 binding to the cell surface or by its degradation, as previously reported (55-58). MMP-2 can activate MMP-9 and MMP-13 (43). MMP-13 is secreted from stimulated synoviocytes and fibroblasts, as well as from tumor cells, and plays a critical role in degenerative bone diseases, such as osteoarthritis and rheumatoid arthritis $(59,60)$. As the cardiac valvular complex and cartilage have common structural features, it is conceivable that periostin induces
MMP-13 expression in VICs. To our knowledge, this is the first report to describe the induction of MMPs by periostin. Periostin has been shown to promote collagen fibrillogenesis in myocardial infarction and the cardiac valve explants $(37,61)$, whereas MMPs are involved in the initiation and progression of collagen fibril growth (62). Therefore, we assume that the activation of MMPs mediates, at least in part, the effects of periostin on collagen fibrillogenesis and angiogenesis. A recent study has indicated that periostin is induced during LV remodeling after cardiac injury without affecting cardiac fibroblast proliferation (35). Interestingly, we also found that periostin was reexpressed during cardiac valve degeneration without affecting VIC proliferation, which suggests that it regulates cardiac pathologic remodeling via common biological mechanisms, including those that involve MMPs. Taken together, our data demonstrate that periostin causes cardiac valve complex degeneration by promoting angiogenesis and production of MMPs, which in turn facilitate the infiltration of periostin- 
A

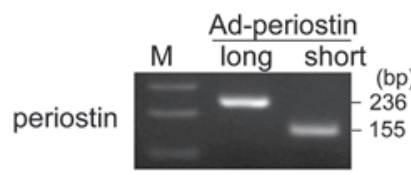

IB:

periostin

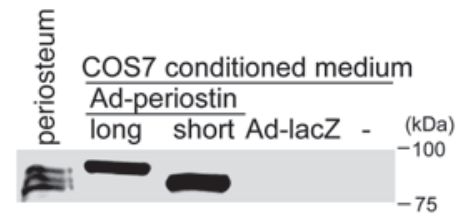

B
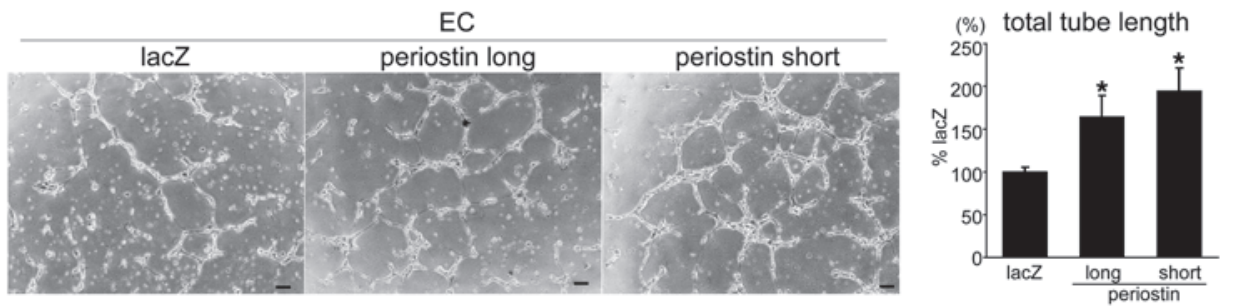

C
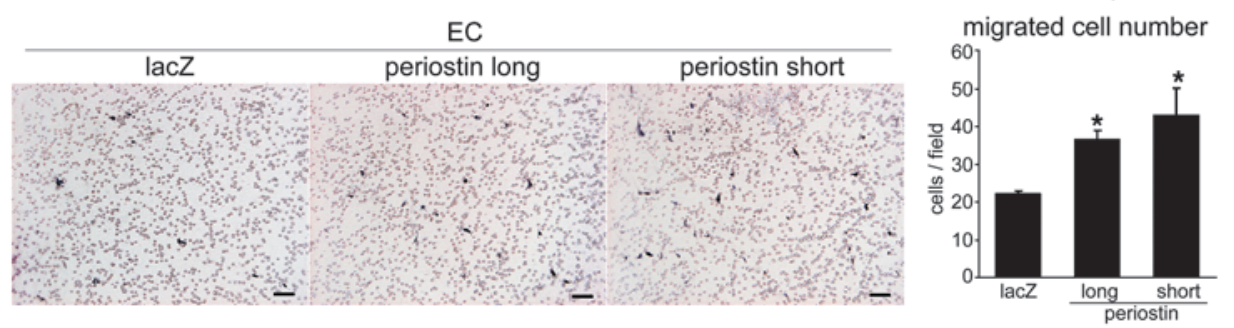

D

EC
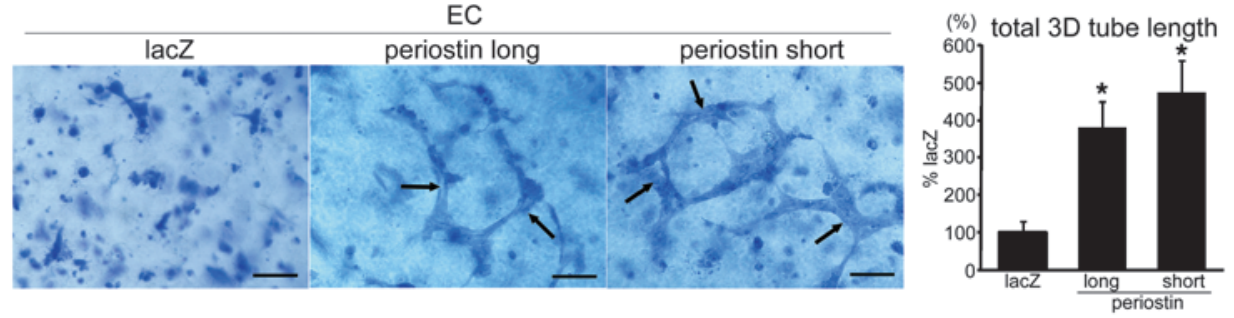

E
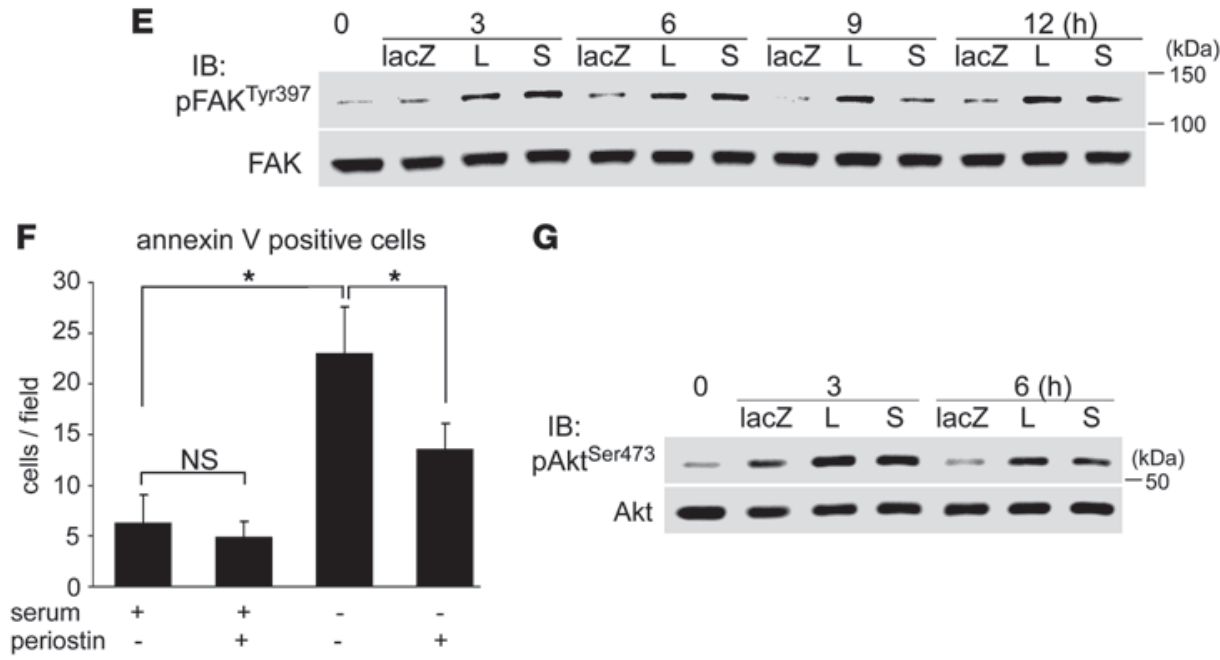

G

H BrdU positive cells
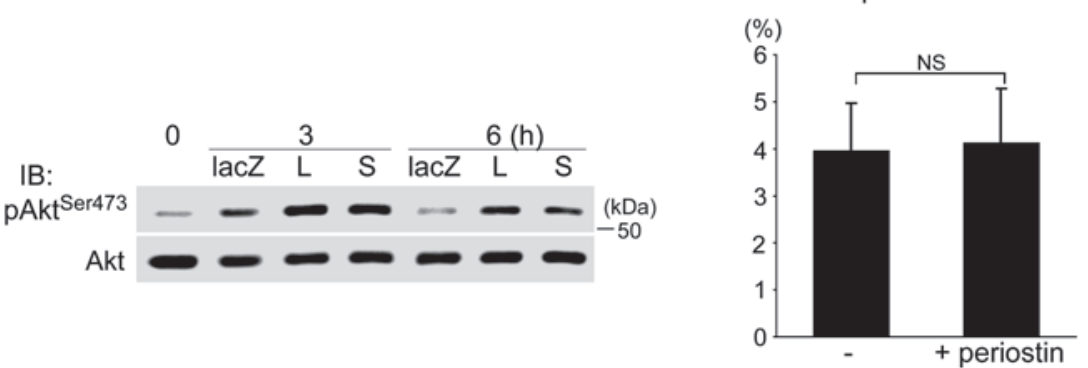

Figure 7

Periostin isoforms comparably promote angiogenesis in ECs in vitro. (A) Generation of isoform-specific murine periostin. Left: PCR of DNA from adenoviruses that contain the long or short periostin isoform. Primer pair 2 (see Figure 1A) was used. Right: Western blot analysis of COS7 cell-conditioned media. COS7 cells were infected with adenoviruses (Ad) that carried the long or short isoform of periostin or LacZ, and the conditioned media were collected. (B-D) Tube formation assay (B), migration assay (C), and 3D vasculogenesis assay (D) for human coronary artery ECs. ECs were stimulated with COS7-conditioned media, prepared as described in A. Both the long and short isoforms of periostin significantly and comparably promoted tube formation and mobilization. The formed luminal structures are shown by arrows in $\mathbf{D}$. Scale bars: $100 \mu \mathrm{m}$. ${ }^{*} P<0.05$ versus LacZ. (E) FAK activation in ECs by periostin stimulation. L, long periostin isoform; S, short periostin isoform. (F) Apoptosis assay for ECs. ECs were stimulated with or without the long isoform of periostin either in $10 \% \mathrm{FBS}$ or under serum starvation conditions for 24 hours. Periostin significantly inhibited serum starvation-induced EC apoptosis. ${ }^{*} P<0.05$. (G) Periostin-induced Akt activation in ECs. (H) BrdU incorporation assay for ECs stimulated with or without the long isoform of periostin. Periostin did not affect EC proliferation. 
A
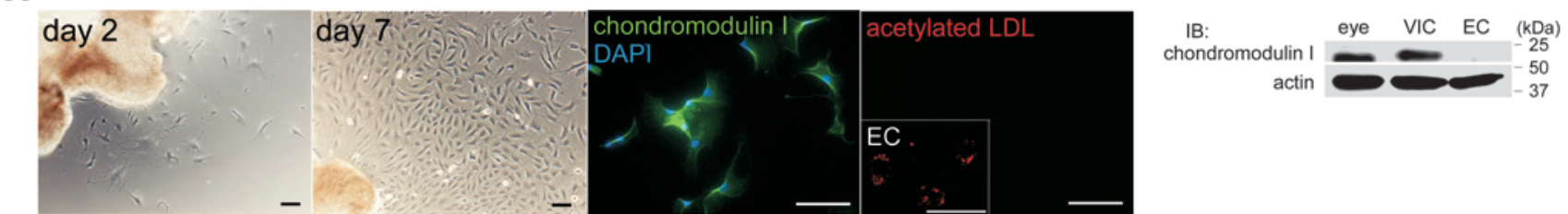

B
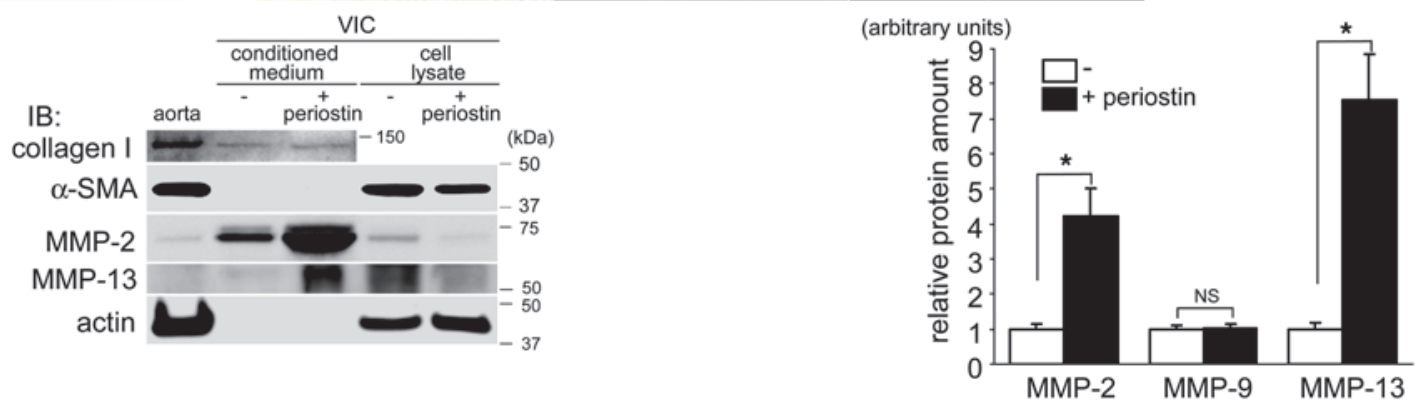

C
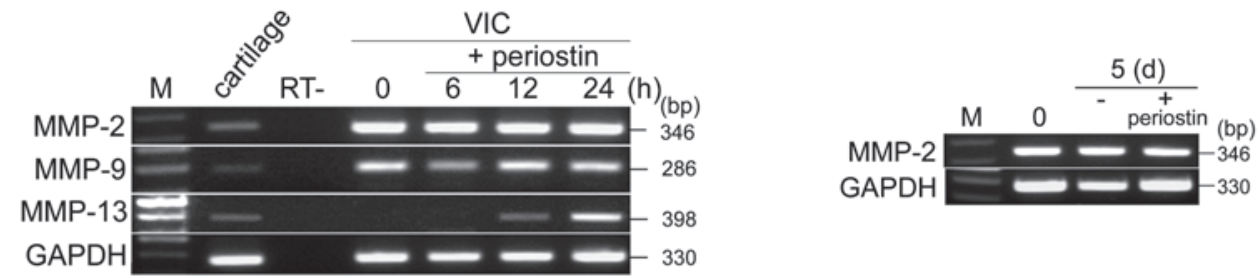

D
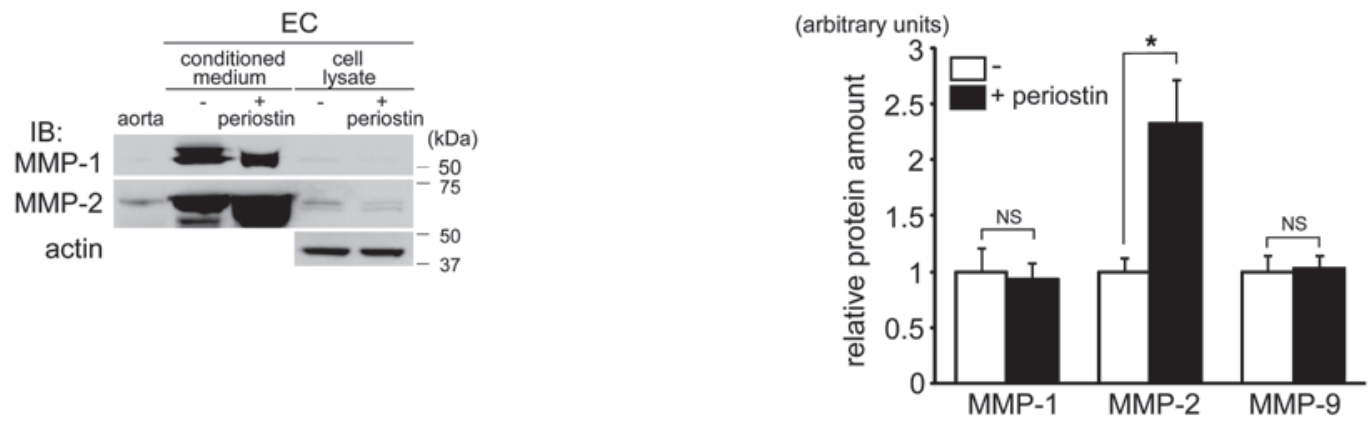

E
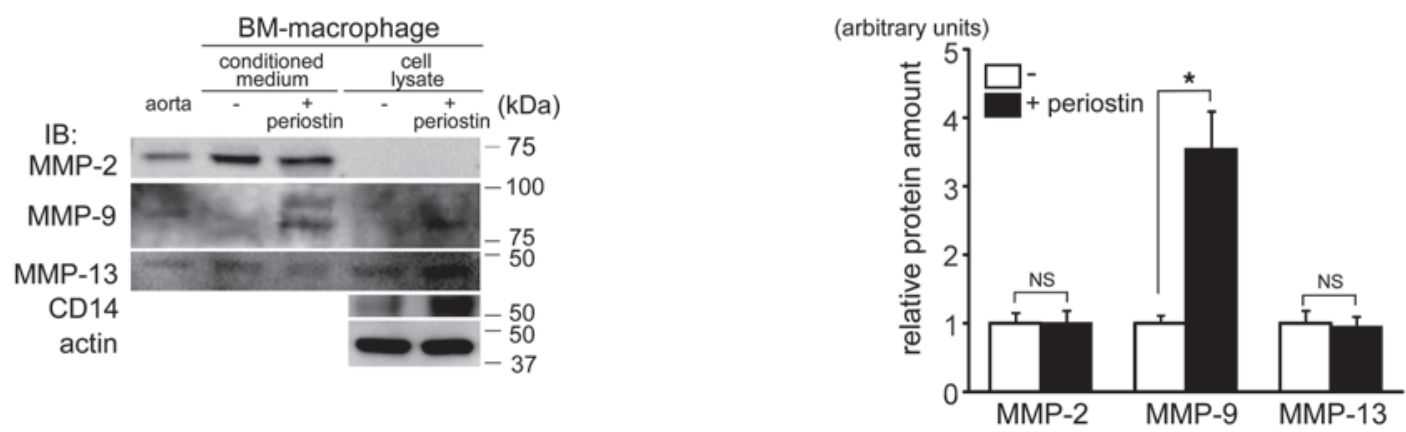

\section{Figure 8}

Periostin induces the secretion of MMPs from VICs, ECs, and macrophages in vitro. (A) Light microscopy, immunofluorescence staining, and Western blot analysis of cultured rat VICs. The VICs were fibroblast-like in appearance and expressed chondromodulin I, but did not express acetylated LDL. The ECs in the inset and the rat eye in the Western blot served as positive controls. Scale bars: $100 \mu \mathrm{m}$. (B) Expression and secretion of collagen I, $\alpha$-SMA, MMP-2, and MMP-13 by VICs following periostin stimulation. Conditioned media and cell lysates were obtained from VICs with or without periostin stimulation and subjected to Western blot analysis. Periostin prominently stimulated secretion of MMP-2 and MMP-13 by VICs, whereas collagen I production and $\alpha$-SMA expression were unchanged. Densitometric quantitative analysis of MMP levels in the conditioned media from the VIC cultures is also shown. (C) RT-PCR analysis of MMP expression in VICs after periostin stimulation. Periostin increased the transcription of $\mathrm{Mmp13}$, but not that of $\mathrm{Mmp2}$ or Mmp9. Cartilage served as a positive control. Also shown is Mmp2 mRNA expression in VICs after stimulation with or without periostin for 5 days. (D and E) Expression and secretion of MMPs by human coronary artery ECs (D) and cultured mouse BM-derived macrophages (E) following periostin stimulation. Densitometric quantitative analyses of MMP levels in the conditioned media from cultures of ECs and macrophages is also shown. Periostin significantly induced secretion of MMP-2 and MMP-9 from ECs and macrophages, respectively. ${ }^{\star} P<0.05$. 


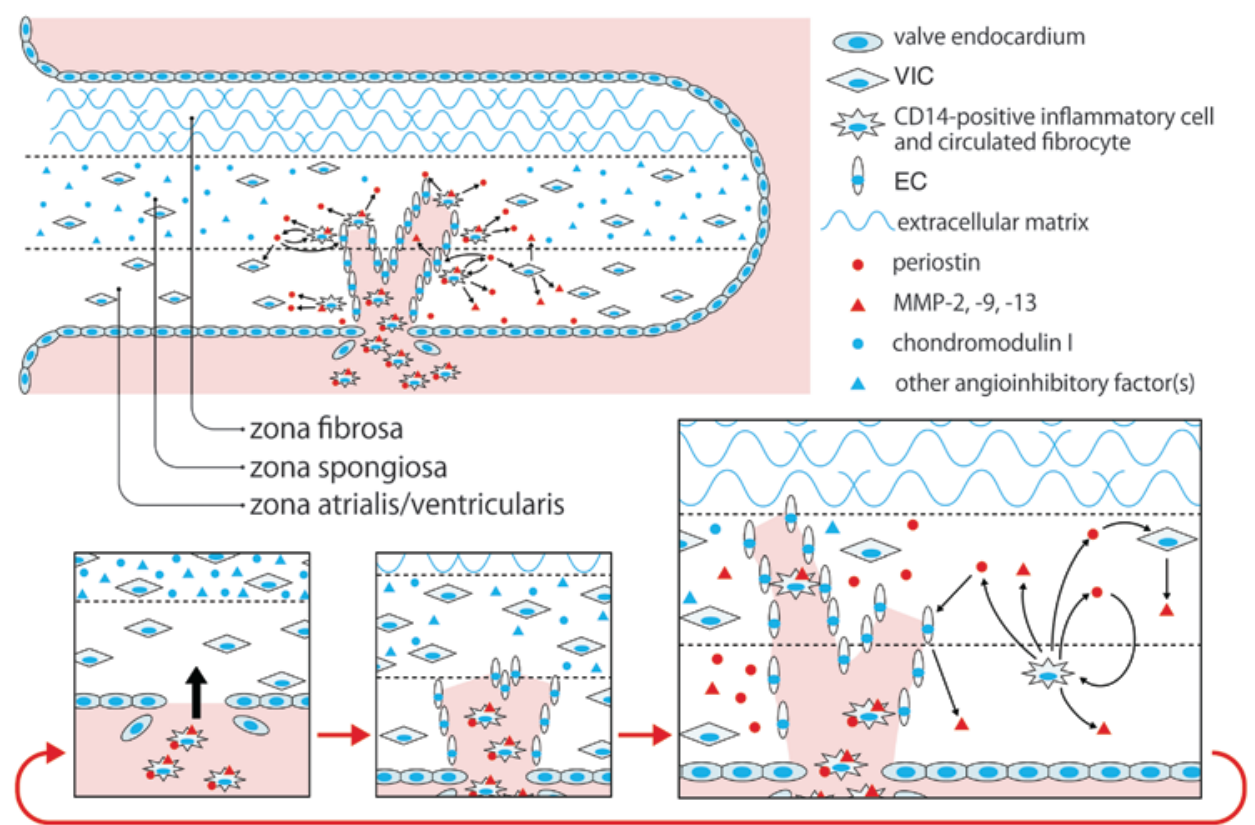

Figure 9

Conceptual framework for the roles of periostin and chondromodulin I in the progression of atherosclerotic and rheumatic VHD. The circulating CD14-positive inflammatory cells (and possibly fibrocytes) that express periostin initially infiltrate the zona atrialis/ventricularis of the cardiac valve. Thereafter, periostin secreted from these cells not only promotes angiogenesis by induction of EC migration and inhibition of EC apoptosis, but also stimulates the production of MMP-2, MMP-9, and MMP-13 by ECs, VICs, and engrafted macrophages, which advance cardiac valve degeneration. Valve degeneration in turn facilitates the infiltration of circulating periostin-expressing cells, thereby creating a vicious circle. The origin of the engrafted ECs is unknown; it is possible that periostin enhances the recruitment of circulating endothelial progenitor cells into the valves or promotes the penetration of microvessels from the annulus region or roots into the leaflets. In contrast, chondromodulin I blocks angiogenesis through inhibition of EC migration and induction of EC apoptosis, although other angioinhibitory factors are involved during the earlier stages of valve degeneration. Therefore, the equilibrium between periostin and chondromodulin I (as well as other angioinhibitory factors) defines the progression of atherosclerotic and rheumatic cardiac valve degeneration by controlling angiogenesis and MMP production.

expressing exogenous inflammatory cells and myofibroblasts, thereby creating a vicious circle in degenerative VHD (Figure 9).

The HF diet significantly induced the expression of periostin, $\alpha$-SMA, MMP-2, and MMP-13 in the cardiac valve complexes of WT mice, although the underlying mechanism remains unclear. Because the periostin promoter contains a site for activator protein-1 (AP-1) and a Smad binding element (63) and is induced by various cellular stresses (64), we hypothesize that the upregulation of periostin expression is due to periostin induction and/or the accumulation of exogenous myofibroblasts and inflammatory cells as a result of HF diet-provoked oxidative stress and inflammation.

The HF diet increased body weights and serum total cholesterol levels by 1.8-fold in both WT and Postn- ${ }^{-1-}$ mice, reflecting the increases observed clinically in humans. Surprisingly, degeneration and the expression of $\alpha$-SMA, MMP-2, and MMP-13 in the cardiac valve complex were strikingly diminished in the Postn-/- mice. The detailed mechanisms underlying this phenotypic change are not elucidated by this experiment, given the limitation that the role of periostin in specific cell types is not demonstrable in the germline knockout mice used. However, it is possible that these findings reflect the decreased abilities of myofibroblasts and inflammatory cells to adhere to and infiltrate the valves of Postn ${ }^{-1-}$ mice. In support of this notion, a recent study showed that the number of
$\alpha$-SMA-positive cells was significantly lower in the infarct areas of Postn ${ }^{-1-}$ mice than in those of WT mice and that adenovirus-mediated Postn gene transfer into the knockout mice restored the number of $\alpha$-SMA-positive cells to the levels seen in WT mice (37).

In the present study, we focused on the pathophysiologic functions of periostin in degenerative atherosclerotic and rheumatic VHD and found that periostin is a critical factor in the degeneration of the cardiac valve complex. The therapeutic effect of HMG CoA reductase inhibitors on aortic valve stenosis is currently a controversial issue. Therefore, periostin may represent a novel therapeutic target to prevent the progression of atherosclerotic and rheumatic VHD, including aortic valve stenosis. Further studies are required to address this issue.

\section{Methods}

Animals. WT ICR mice and Wistar rats were purchased from Japan CLEA. The genetic backgrounds of the Postn ${ }^{-/}$ mice (37) and the Lect1 ${ }^{-/-}$mice (23) have been described previously. The offspring were genotyped by either Southern blotting or PCR, and WT littermates of these knockout strains were used as controls. All experimental procedures and protocols were approved by the Animal Care and Use Committee of Keio University and conformed to NIH guidelines for the care and use of laboratory animals.

Human samples. Samples of 10 aortic and 11 mitral valves were collected from patients ( 9 males and 12 females; average age, $63.9 \pm 12.0$ years) who were undergoing valve replacement surgery due to valvular stenosis or regurgitation. The average ages of the patients with atherosclerotic valves $(n=8)$, rheumatic valves $(n=9)$, and valvular prolapse $(n=4)$ were $70.0 \pm 9.2$ years, $64.7 \pm 7.8$ years, and $50.0 \pm 15.3$ years, respectively. Specimens were fixed in formaldehyde immediately after removal and then embedded in paraffin. As controls, 12 microscopically and macroscopically normal, noncalcified, smooth, and pliable valves ( 7 aortic and 5 mitral valves) were collected from 10 autopsied patients ( 8 males and 2 females; average age, $65.2 \pm 5.8$ years) who died of noncardiac diseases. The use of autopsied and surgical specimens of human tissues was approved by the institutional review board of Keio University. All participants provided informed consent for their tissue to be used.

RT-PCR. Total RNA was prepared with the TRIzol reagent (Gibco; Invitrogen). RT-PCR was performed as described previously (23) using the following primers: mouse Postn forward, $5^{\prime}$-AACCAAGGACCTGAAACACG-3'; mouse Postn reverse, 5'-CAAAGAGCGTGAAGTGACCA-3'; mouse Postn primer pair 1 forward, 5'-GATAAAATACATCCAAATCAAGTTTGTTCG-3'; mouse Postn primer pair 1 reverse, 5'-AAACTCTGTGGTCTGGCCTCTGGG-3'; mouse Postn primer pair 2 forward, 5'-GATAAAATA- 
CATCCAAATCAAGTTTGTTCG-3'; mouse Postn primer pair 2 reverse, 5'-CGTGGATCACTTCTGTCACCGTTTCGC-3'; mouse Lect1 forward 1, 5'-TTGGTTGATGCTTCAGTGTG-3'; mouse Lect1 forward 2, 5'CCGCTTCCTCGTGCTTTACGG-3'; mouse Lect 1 reverse, 5' -CTTGTGCACAGACCAGAACAA- $3^{\prime}$; mouse GAPDH forward, 5' -TTCAACGGCACAGTCAAGG-3'; mouse GAPDH reverse, 5'-CATGGACTGTGGTCATGAG-3'; rat Mmp2 forward, 5'-ACACTGGGACCTGTCACTCC-3'; rat $M m p 2$ reverse, 5'-TCCAGTTAAAGGCAGCGTCT-3'; rat Mmp9 forward, 5'-CACTGTAACTGGGGGCAACT-3'; rat Mmp9 reverse, 5'-AGGGGAGTCCTCGTGGTAGT-3'; rat Mmp13 forward, 5'-TGTGGCTGGCTTTACATTTG-3'; rat Mmp13 reverse, 5'-ACATGGAGGAGCATGAAAGG-3'; rat Lect1 forward, 5'-AAGCAGTGCTCCCTCTACCA-3'; rat Lect1 reverse, 5'-AATTCTTGCTTGGCAGTGCT-3'.

Western blotting. Western blot analysis was performed as described previously (23). To obtain cell lysates, murine cardiac valves were homogenized, then concentrated using a Microcon centrifugal ultrafiltration filter (Millipore). The primary antibodies used were as follows: anti-mouse periostin antibody (directed against the C-terminus of periostin and recognizing both the long and short isoforms; ref. 37); anti-periostin (provided by R. Markwald, Medical University of South Carolina, Charleston, South Carolina, USA); anti- $\alpha$-SMA (Sigma-Aldrich); anti-collagen type I (C-18; Santa Cruz Biotechnology); anti-phospho-FAK (Tyr397; Cell Signaling Technology); anti-FAK (Cell Signaling Technology); anti-phospho-Akt (Ser473; Cell Signaling Technology); anti-Akt (Cell Signaling Technology); anti-MMP-1 (SA-102; BIOMOL Research Laboratories); anti-MMP-2 (42-5D11; Daiichi Fine Chemical); anti-MMP-9 (C-20; Santa Cruz Biotechnology); and antiMMP-13 (AB8120; Chemicon International). The level of total protein was assessed by reblotting with anti-actin antibody (C-11; Santa Cruz Biotechnology) or by staining with Ponceau-S (Sigma-Aldrich).

Histology, immunohistochemistry, and immunofluorescence staining. Mouse and rat specimens were perfused from the apex with $4 \%$ paraformaldehyde, fixed overnight, and then embedded in paraffin; histology and immunostaining were performed as previously described (23). Some samples were embedded in OCT compound (Sakura Finetek Japan) after fixation, and then frozen in liquid nitrogen. Primary antibodies were as follows: anti-periostin (provided by R. Markwald; for triple immunofluorescence, MAB3548; R\&D Systems); anti-periostin antibody 1 (RD181045050; BioVendor); anti-periostin antibody 2 (directed against the $\mathrm{N}$-terminus of human periostin); anti-chondromodulin I (23); anti-vWF (A0 and R7; Thermo Scientific); anti-VEGF (A-20; Santa Cruz Biotechnology); anti-collagen type I (Rockland); anti-elastin (Elastin Products); anti-vimentin (GP53; PROGEN Biotechnik); anti-CD14 (M-305; Santa Cruz Biotechnology); anti-cadherin 11 (5B2H5; Invitrogen); and anti-NFATc1 (7A6; BD Biosciences - Pharmingen). The M.O.M. Immunodetection Kit (Vector Laboratories) was used to detect the mouse primary antibody used to blot the murine tissues.

For IHC, antigen unmasking was performed by heating in a microwave oven the sections in $10 \mathrm{mM}$ citric acid monohydrate ( $\mathrm{pH}$ 6.0; DAKO) for 3 minutes. Immunohistochemical signals were then detected using the DakoCytomation EnVision+ System-HRP (DAB) Kit (DAKO) or the Universal LSAB+ Kit/HRP for Mouse/Rabbit/Goat (DAKO). Percentages of stained areas in the sections were analyzed using NIH Image J software version 1.37.

For immunofluorescence analyses, sections were incubated with secondary antibodies conjugated with Alexa Fluor 488 or Alexa Fluor 546 (Invitrogen). The slides were observed under a confocal laser-scanning microscope (LSM 510 META; Carl Zeiss) or an immunofluorescence microscope (BIOREVO BZ-9000; Keyence). As a negative control in each immunostaining experiment, nonimmune rabbit serum was substituted for the primary antibody.

Feeding protocol. Postn ${ }^{-/-}$mice and their WT littermates at 12 weeks of age were fed either normal diet CE-2 (Japan CLEA) or High Fat Diet 32 (Japan CLEA) for 4 months, and were then subjected to analysis. In the
HF diet, $57 \%$ of the total calories are derived from fat, compared with $10 \%$ in the normal diet.

Echocardiography. Transthoracic echocardiography was performed on WT and Post $n^{-/-}$mice ( $n=8$ per group) using a Vevo 770 echocardiogram (VisualSonics) equipped with a $45-\mathrm{MHz}$ sector-array transducer. Mice were anesthetized with isoflurane inhalation, and the hearts were scanned at a rate of 100 frames/s using 2D mode or M-mode. The high-echogenic areas of the aortic and mitral valve annuli were traced using the $2 \mathrm{D}$ mode of the long axis view. The aortic valve thickness, the thickness of the interventricular septum and LV posterior wall, and the end-diastolic and end-systolic diameters of the LV were measured using the M-mode of the long axis view.

Preparation of adenovirus. Construction of adenoviruses that express either the long or the short isoform of mouse periostin or nuclear localization signal-LacZ was performed with the Adeno-X Expression System 2 (BD Biosciences), as described previously (37). COS7 cells were infected with these adenoviruses in serum-free DMEM, and the conditioned media were collected.

Isolation and primary culture of adult rat VICs and mouse BM-derived macrophages. The isolation and primary culture of VICs were performed according to the previously described protocol (23), with minor modifications. The valvular pieces cut by a blade were grown in M199 medium (SigmaAldrich) with $50 \% \mathrm{FBS}$ for 24 hours at $37^{\circ} \mathrm{C}$. Medium was then replaced with M199 that contained 10\% FBS.

Mouse BM-derived macrophages were isolated as previously described (65). Briefly, the femur and tibia were excised from the mice, cut at both ends, and flushed with the medium using a syringe. The BM cells were plated and cultured for 7 days at a concentration of $2 \times 10^{6}$ cells $/ \mathrm{ml}$ in RPMI 1640 GlutaMAX I medium (Invitrogen) supplemented with 10\% FBS, 1\% penicillin-streptomycin, and $50 \mu \mathrm{g} / \mathrm{ml}$ recombinant human M-CSF (PeproTech). Cells were washed with PBS, and the medium was changed every 3 days. More than $95 \%$ of the cells were identified as macrophages based on nonspecific esterase staining.

Cell culture. Human coronary artery ECs were purchased from Takara Biotechnology, maintained as described previously (23), and used at passages 3-5. MC3T3-E1 cells (ATCC) were purchased and maintained according to the manufacturer's instructions. In some experiments, VICs and ECs were treated with $10 \mu \mathrm{g} / \mathrm{ml}$ DiI-conjugated acetylated LDL (Invitrogen) for 1 hour at $37^{\circ} \mathrm{C}$ and observed under an immunofluorescence microscope. For the MMP production analyses, VICs, coronary artery ECs, and BM-derived macrophages were serum-starved and stimulated for 7 days (VICs) or 2 days (ECs and macrophages) with $5 \mu \mathrm{g} / \mathrm{ml}$ recombinant human periostin (R\&D Systems), which corresponds to the long isoform of periostin, and the conditioned media and cell lysates were collected. In some experiments, BM-derived macrophages were stimulated with or without $50 \mu \mathrm{M} \mathrm{H}_{2} \mathrm{O}_{2}$ (Wako), $10 \mathrm{ng} / \mathrm{ml} \mathrm{recombinant}$ human TNF- $\alpha$ (R\&D Systems), $10 \mu \mathrm{g} / \mathrm{ml}$ LPS (from E. coli 0111:B4; SigmaAldrich), or $10 \mathrm{ng} / \mathrm{ml}$ human TGF- $\beta_{1}$ (R\&D Systems) for 24 hours.

Tube formation assay. A tube formation assay using ECs was performed as described previously (23), with minor modifications. After incubation for 4 hours under starvation conditions, the ECs were trypsinized and suspended in conditioned medium, which contained the long or short periostin isoform or LacZ. The assays were performed with 6 replicates.

Cell migration assay. The cell migration assay was performed as described previously (23), with minor modifications. ECs were cultured in modified Boyden chambers with 8 - $\mu \mathrm{m}$-pore filter inserts (Chemotaxicell; Kurabo) for 24-well plates (BD Labware). The conditioned medium was placed in the lower chamber, and ECs were seeded $\left(5 \times 10^{4}\right.$ cells per well) into the upper chamber under serum-free conditions. The assays were performed with 6 replicates.

In vitro $3 D$ vasculogenesis assay. The $3 \mathrm{D}$ vasculogenesis assay for $\mathrm{ECs}$ was performed according to a previously described protocol (66), with minor modifications. Rat tail collagen (High Concentration, type 1; BD Biosci- 
ences), $1 \mathrm{~N} \mathrm{NaOH}$, and serum-free EBM-2 (Lonza) with or without periostin were mixed on ice to prepare the collagen I solution. The ECs were trypsinized and added to the collagen solution to produce the EC-collagen mixture $\left(1 \times 10^{6}\right.$ cells $/ \mathrm{ml}$ and $3 \mathrm{mg} / \mathrm{ml}$ collagen). Aliquots $(50 \mu \mathrm{l})$ of the mixture were placed in the wells of a 96-well plate and allowed to gel at $37^{\circ} \mathrm{C}$ for 30 minutes. EBM-2 supplemented with $0.5 \mathrm{mg} / \mathrm{ml} \mathrm{BSA}$ and $1 \%$ ITS Liquid Media Supplement (Sigma-Aldrich) was added to each well and incubated at $37^{\circ} \mathrm{C}$ for 24 hours. The cells were fixed with $2 \%$ PFA overnight, stained with $0.05 \%$ toluidine blue solution (Muto Pure Chemicals) for 45 minutes, washed with water for 2 hours, and observed under a phasecontrast microscope. The assays were performed with 6 replicates.

Cell proliferation assay. The effects of periostin on EC and VIC proliferation were examined using the BrdU Labeling and Detection Kit I (Roche Diagnostics) according to the manufacturer's instructions. Cells were grown to $50 \%$ confluence, and then starved for 4 hours (ECs) or 24 hours (VICs). The cells were then incubated with $10 \mu \mathrm{M}$ BrdU for 2 hours and subjected to immunostaining. The fluorescent cells in 4 random fields per well were counted under an immunofluorescence microscope. The assays were performed with 5 replicates.

Apoptosis assay. The annexin V-FITC Apoptosis Detection Kit (BioVision) was used according to the manufacturer's instructions. ECs or VICs were cultured in medium with or without periostin. The cells were then labeled with annexin V-FITC and propidium iodide, washed with PBS, and fixed with $2 \%$ formaldehyde for 15 minutes. The annexin $\mathrm{V}$-positive, propidium iodide-negative cells in 4 random fields per well were counted under an immunofluorescence microscope. The assays were performed with 6 replicates.

Ex vivo aortic ring angiogenesis assay. The aortic ring assay was performed as previously described (65). Briefly, the aortic roots and annuli just above the aortic valves were dissected from WT and Postn ${ }^{-/}$mice, cleaned of blood and periaortic fibroadipose tissue, and sectioned into $1-\mathrm{mm}$ rings with a scalpel. The aortic root rings were embedded in $2 \mathrm{mg} / \mathrm{ml}$ collagen I gel so that the luminal axis lay parallel to the bottom of the well. The rings were cultured in serum-free EBM- 2 in 96 -well plates at $37^{\circ} \mathrm{C}$, and the medium was replaced every 3 days. The microvessels that radiated outward from the aortic root were counted at the indicated time points under a phase-contrast microscope. In some experiments, $5 \mu \mathrm{g} / \mathrm{ml}$ recombinant human periostin was added to the medium. The assays were performed with 6 replicates.

Statistics. All results are presented as mean \pm SD. Statistical significance was evaluated with the unpaired 2-tailed Student's $t$ test for comparisons between 2 mean values. Multiple comparisons of more than 3 groups were performed by ANOVA. $P$ values of less than 0.05 were considered statistically significant.

\section{Acknowledgments}

We thank Yoshiko Miyake for expert technical assistance with the histology. This study received support from the program for the Promotion of Fundamental Studies in Health Science of the National Institute of Biomedical Innovation (NIBIO; to K. Fukuda), Grants-in-Aid for Scientific Research from the Ministry of Education, Culture, Sports, Science, and Technology of Japan (to D. Hakuno and K. Fukuda), a Japan Heart Foundation/Novartis Grant for Research Award on Molecular and Cellular Cardiology (to D. Hakuno), and a grant from the Keio Gijuku Fund for the Advancement of Education and Research (to D. Hakuno).

Received for publication August 27, 2009, and accepted in revised form April 21, 2010.

Address correspondence to: Keiichi Fukuda, Department of Regenerative Medicine and Advanced Cardiac Therapeutics, Keio University School of Medicine, 35 Shinanomachi, Shinjuku-ku, Tokyo 160-8582, Japan. Phone: 81.3.5363.3874; Fax: 81.3.5363.3875; E-mail:kfukuda@sc.itc.keio.ac.jp.

Daihiko Hakuno's present address is: Cardiovascular Division, Department of Internal Medicine, National Defense Medical College, Saitama, Japan.
1. Nkomo VT, Gardin JM, Skelton TN, Gottdiener JS, Scott CG, Enriquez-Sarano M. Burden of valvular heart diseases: a population-based study. Lancet. 2006;368(9540):1005-1011

2. Mohler ER 3rd, Gannon F, Reynolds C, Zimmerman R, Keane MG, Kaplan FS. Bone formation and inflammation in cardiac valves. Circulation. 2001;103(11):1522-1528.

3. Freeman RV, Otto CM. Spectrum of calcific aortic valve disease: pathogenesis, disease progression, and treatment strategies. Circulation. 2005;111(24):3316-3326.

4. Aikawa E, et al. Multimodality molecular imaging identifies proteolytic and osteogenic activities in early aortic valve disease. Circulation. 2007;115(3):377-386

5. Rajamannan NM, Bonow RO, Rahimtoola SH. Calcific aortic stenosis: an update. Nat Clin Pract Cardiovasc Med. 2007;4(5):254-262.

6. Cowell SJ, et al. A randomized trial of intensive lipid-lowering therapy in calcific aortic stenosis. NEngl J Med. 2005;352(23):2389-2397.

7. Rossebo $A B$, et al. Intensive lipid lowering with simvastatin and ezetimibe in aortic stenosis. $N$ Engl J Med. 2008;359(13):1343-1356.

8 . Satta J, et al. Progression of human aortic valve stenosis is associated with tenascin-C expression. J Am Coll Cardiol. 2002;39(1):96-101.

9. Caira FC, et al. Human degenerative valve disease is associated with up-regulation of low-density lipoprotein receptor-related protein 5 receptor-mediated bone formation. J Am Coll Cardiol. 2006;47(8):1707-1712.
10. Walker GA, Masters KS, Shah DN, Anseth KS, Leinwand LA. Valvular myofibroblast activation by transforming growth factor-beta: implications for pathological extracellular matrix remodeling in heart valve disease. Circ Res. 2004;95(3):253-260.

11. Kaden JJ, et al. Receptor activator of nuclear factor kappaB ligand and osteoprotegerin regulate aortic valve calcification. J Mol Cell Cardiol. 2004;36(1):57-66.

12. Osman L, et al. A novel role of the sympatho-adrenergic system in regulating valve calcification. Circulation. 2007;116(11 suppl):I282-I287.

13. Hakuno D, Kimura N, Yoshioka M, Fukuda K. Molecular mechanisms underlying the onset of degenerative aortic valve disease. $\mathrm{J} \mathrm{Mol} \mathrm{Med}$. 2009;87(1):17-24.

14. Galvin KM, et al. A role for smad6 in development and homeostasis of the cardiovascular system. Nat Genet. 2000;24(2):171-174.

15. Hanada K, et al. Perturbations of vascular homeostasis and aortic valve abnormalities in fibulin-4 deficient mice. Circ Res. 2007;100(5):738-746.

16. Garg V, et al. Mutations in NOTCH1 cause aortic valve disease. Nature. 2005;437(7056):270-274.

17. Hammon JW Jr, O'Sullivan MJ, Oury J, Fosburg RG. Allograft cardiac valves. A view through the scanning electron microscope. J Thorac Cardiovasc Surg. 1974;68(3):352-360.

18. Millington-Sanders C, Meir A, Lawrence L, Stolinski C. Structure of chordae tendineae in the left ventricle of the human heart. J Anat. 1998; 192(pt 4):573-581.

19. Shukunami C, Oshima Y, Hiraki Y. Chondromod-
ulin-I and tenomodulin: a new class of tissue-specific angiogenesis inhibitors found in hypovascular connective tissues. Biochem Biophys Res Commun. 2005;333(2):299-307.

20. Ashraf S, Walsh DA. Angiogenesis in osteoarthritis. Curr Opin Rheumatol. 2008;20(5):573-580.

21. Soini Y, Salo T, Satta J. Angiogenesis is involved in the pathogenesis of nonrheumatic aortic valve stenosis. Hum Pathol. 2003;34(8):756-763.

22. Rajamannan NM, et al. Calcified rheumatic valve neoangiogenesis is associated with vascular endothelial growth factor expression and osteoblast-like bone formation. Circulation. 2005;111(24):3296-3301.

23. Yoshioka M, et al. Chondromodulin-I maintains cardiac valvular function by preventing angiogenesis. Nat Med. 2006;12(10):1151-1159.

24. Kimura N, et al. Local tenomodulin absence, angiogenesis, and matrix metalloproteinase activation are associated with the rupture of the chordae tendineae cordis. Circulation. 2008;118(17):1737-1747.

25. Takeshita S, Kikuno R, Tezuka K, Amann E. Osteoblast-specific factor 2: cloning of a putative bone adhesion protein with homology with the insect protein fasciclin I. Biochem J. 1993;294(pt 1):271-278.

26. Horiuchi $\mathrm{K}$, et al. Identification and characterization of a novel protein, periostin, with restricted expression to periosteum and periodontal ligament and increased expression by transforming growth factor beta. J Bone Miner Res. 1999;14(7):1239-1249.

27. Zinn K, McAllister L, Goodman CS. Sequence analysis and neuronal expression of fasciclin I in grasshopper and Drosophila. Cell. 1988;53(4):577-587. 
28. Gillan L, Matei D, Fishman DA, Gerbin CS, Karlan BY, Chang DD. Periostin secreted by epithelial ovarian carcinoma is a ligand for alpha(V)beta(3) and alpha(V)beta(5) integrins and promotes cell motility. Cancer Res. 2002;62(18):5358-5364.

29 . Bao S, et al. Periostin potently promotes metastatic growth of colon cancer by augmenting cell survival via the Akt/PKB pathway. Cancer Cell. 2004;5(4):329-339.

30. Lindner V, Wang Q, Conley BA, Friesel RE, Vary CP. Vascular injury induces expression of periostin: implications for vascular cell differentiation and migration. Arterioscler Thromb Vasc Biol. 2005;25(1):77-83.

31. Roy S, et al. Transcriptome-wide analysis of blood vessels laser captured from human skin and chronic wound-edge tissue. Proc Natl Acad Sci U S A. 2007;104(36):14472-14477.

32. Kruzynska-Frejtag A, Machnicki M, Rogers R, Markwald RR, Conway SJ. Periostin (an osteoblastspecific factor) is expressed within the embryonic mouse heart during valve formation. Mech Dev. 2001;103(1-2):183-188.

33. Katsuragi N, et al. Periostin as a novel factor responsible for ventricular dilation. Circulation. 2004;110(13):1806-1813.

34. Oka T, et al. Genetic manipulation of periostin expression reveals a role in cardiac hypertrophy and ventricular remodeling. Circ Res. 2007;101(3):313-321.

35. Lorts A, Schwanekamp JA, Elrod JW, Sargent MA, Molkentin JD. Genetic manipulation of periostin expression in the heart does not affect myocyte content, cell cycle activity, or cardiac repair. Circ Res. 2009;104(1):e1-e7.

36. Stanton LW, et al. Altered patterns of gene expression in response to myocardial infarction. Circ Res. 2000;86(9):939-945.

37. Shimazaki $M$, et al. Periostin is essential for cardiac healing after acute myocardial infarction. $J$ Exp Med. 2008;205(2):295-303.

38. Butcher JT, Norris RA, Hoffman S, Mjaatvedt CH, Markwald RR. Periostin promotes atrioventricular mesenchyme matrix invasion and remodeling mediated by integrin signaling through Rho/PI 3 kinase. Dev Biol. 2007;302(1):256-266.

39. Snider $\mathrm{P}$, et al. Periostin is required for maturation and extracellular matrix stabilization of noncardiomyocyte lineages of the heart. Circ Res. 2008;102(7):752-760.

40. Lincoln J, Lange AW, Yutzey KE. Hearts and bones: shared regulatory mechanisms in heart valve, cartilage, tendon, and bone development. Dev Biol.
2006;294(2):292-302.

41. GetzGS, Reardon CA. Diet and murine atherosclerosis. Arterioscler Thromb Vasc Biol. 2006;26(2):242-249.

42. Drolet MC, Roussel E, Deshaies Y, Couet J, Arsenaul $\mathrm{M}$. A high fat/high carbohydrate diet induces aortic valve disease in C57BL/6J mice. J Am Coll Cardiol. 2006;47(4):850-855.

43. Visse R, Nagase H. Matrix metalloproteinases and tissue inhibitors of metalloproteinases: structure, function, and biochemistry. Circ Res. 2003;92(8):827-839.

44. Lindsey ML, et al. Selective matrix metalloproteinase inhibition reduces left ventricular remodeling but does not inhibit angiogenesis after myocardial infarction. Circulation. 2002;105(6):753-758.

45. Soini Y, Satta J, Maatta M, Autio-Harmainen H. Expression of MMP-2, MMP9, MT1-MMP, TIMP1, and TIMP2 mRNA in valvular lesions of the heart. J Pathol. 2001;194(2):225-231.

46. Jian B, Jones PL, Li Q, Mohler ER 3rd, Schoen FJ, Levy RJ. Matrix metalloproteinase-2 is associated with tenascin-C in calcific aortic stenosis. $A m J$ Pathol. 2001;159(1):321-327.

47. Rabkin E, Aikawa M, Stone JR, Fukumoto Y, Libby P, Schoen FJ. Activated interstitial myofibroblasts express catabolic enzymes and mediate matrix remodeling in myxomatous heart valves. Circulation. 2001;104(21):2525-2532.

48. Butcher JT, et al. Transcriptional profiles of valvular and vascular endothelial cells reveal phenotypic differences: influence of shear stress. Arterioscler Thromb Vasc Biol. 2006;26(1):69-77.

49. Misfeldt AM, Boyle SC, Tompkins KL, Bautch VL, Labosky PA, Baldwin HS. Endocardial cells are a distinct endothelial lineage derived from Flk1+ multipotent cardiovascular progenitors. Dev Biol. 2009;333(1):78-89

50. Shao R, et al. Acquired expression of periostin by human breast cancers promotes tumor angiogenesis through up-regulation of vascular endothelial growth factor receptor 2 expression. Mol Cell Biol. 2004;24(9):3992-4003.

51. Visconti RP, et al. An in vivo analysis of hematopoietic stem cell potential: hematopoietic origin of cardiac valve interstitial cells. Circ Res. 2006;98(5):690-696.

52. Bucala R. Fibrocytes: Discovery of a Circulating Connective Tissue Cell Progenitor. In: Bucala R, ed. Fibrocytes: New Insights into Tissue Repair and Systemic Fibroses. Toh Tuck Link, Singapore: World Scientific Publishing Company; 2007:1-18.

53. Ferrara N, Davis-Smyth T. The biology of vascular endothelial growth factor. Endocr Rev.
$1997 ; 18(1): 4-25$

54. Siriwardena BS, et al. Periostin is frequently overexpressed and enhances invasion and angiogenesis in oral cancer. Br J Cancer. 2006; 95(10):1396-1403

55. Li YY, McTiernan CF, Feldman AM. Interplay of matrix metalloproteinases, tissue inhibitors of metalloproteinases and their regulators in cardiac matrix remodeling. Cardiovasc Res. 2000;46(2):214-224.

56. Oh J, et al. The membrane-anchored MMP inhibitor RECK is a key regulator of extracellular matrix integrity and angiogenesis. Cell. 2001;107(6):789-800.

57. Munesue S, et al. A novel function of syndecan-2, suppression of matrix metalloproteinase- 2 activation, which causes suppression of metastasis. J Biol Chem. 2007;282(38):28164-28174.

58. Kleiner DE Jr, Tuuttila A, Tryggvason K, StetlerStevenson WG. Stability analysis of latent and active $72-\mathrm{kDa}$ type IV collagenase: the role of tissue inhibitor of metalloproteinases-2 (TIMP-2). Biochemistry. 1993;32(6):1583-1592.

59. Mitchell PG, et al. Cloning, expression, and type II collagenolytic activity of matrix metalloproteinase-13 from human osteoarthritic cartilage. J Clin Invest. 1996;97(3):761-768.

60. Neuhold LA, et al. Postnatal expression in hyaline cartilage of constitutively active human collagenase-3 (MMP-13) induces osteoarthritis in mice. J Clin Invest. 2001;107(1):35-44.

61. Norris RA, et al. Periostin regulates collagen fibrillogenesis and the biomechanical properties of connective tissues. J Cell Biochem. 2007;101(3):695-711.

62. Jung JC, Wang PX, Zhang G, Ezura Y, Fini ME, Birk DE. Collagen fibril growth during chicken tendon development: matrix metalloproteinase- 2 and its activation. Cell Tissue Res. 2009;336(1):79-89.

63. Lindsley A, et al. Identification and characterization of a novel Schwann and outflow tract endocardial cushion lineage-restricted periostin enhancer. Dev Biol. 2007;307(2):340-355.

64. Li G, et al. Phosphatidylinositol-3-kinase signaling mediates vascular smooth muscle cell expression of periostin in vivo and in vitro. Atherosclerosis. 2006;188(2):292-300.

65. Gelati M, Aplin AC, Fogel E, Smith KD, Nicosia RF. The angiogenic response of the aorta to injury and inflammatory cytokines requires macrophages. J Immunol. 2008;181(8):5711-5719.

66. Koh W, Stratman AN, Sacharidou A, Davis GE. In vitro three dimensional collagen matrix models of endothelial lumen formation during vasculogenesis and angiogenesis. Methods Enzymol. 2008;443:83-101. 